\title{
Synthesis of Vitamin K and Related Naphthoquinones Via \\ Demethoxycarbonylative Annulations and a Retro-Wittig Rearrangement
}

\author{
Dipakranjan Mal*, Ketaki Ghosh and Supriti Jana \\ Department of Chemistry, Indian Institute of Technology, Kharagpur, 721302, India \\ dmal@chemiitkgp.ernet.in
}

\section{Content}

1 General $\quad$ S2

2 General procedure for annulation reaction $\quad$ S2

3 NMR data of unknown and some known compounds $\quad$ S2 - S10

4 Scanned spectra of ${ }^{1} \mathrm{H}$ and ${ }^{13} \mathrm{C}$ NMR of unknown compounds $\quad$ S11 - S42

5 References $\quad$ S43 


\section{Experimental Section:}

\section{General}

Melting points are uncorrected. IR spectra were recorded on a Thermo Nicolet Nexus 870 FT-IR spectrophotometer using $\mathrm{KBr}$ pellets. Only the characteristic, strong and medium IR bands are presented. ${ }^{1} \mathrm{H}$ and ${ }^{13} \mathrm{C}$ NMR spectra of the samples in the indicated solvents were recorded on a $200 \mathrm{MHz}$ or $600 \mathrm{MHz}$ spectrometer (Bruker) with residual $\mathrm{CHCl}_{3}$ as the internal standard. Chemical shifts are expressed in $\delta$ unit and coupling constant in Hz. Mass spectra were taken using a VG Autospec M mass spectrometer. Dry solvents used for reactions were purified, before use, according to standard protocols. All solvents for chromatography were distilled prior to use. Columns were prepared with silica gel (60-120 or 230-400 mesh).

\section{General procedure for annulation reaction}

To a stirred solution of LiOBu-t $(3.20 \mathrm{mmol})$ in THF $(40 \mathrm{~mL})$ at $-78{ }^{\circ} \mathrm{C}$ (chloroform/liquid nitrogen bath) under an inert atmosphere was added a solution of a phthalide $(1.00 \mathrm{mmol})$ in THF (5 mL). The resulting yellowish solution was stirred at $-60{ }^{\circ} \mathrm{C}$ for $25 \mathrm{~min}$, after which a solution of a Michael acceptor (1.0-1.5 equiv. unless otherwise stated) in THF (5 mL) was added to it. The cooling bath was removed after about $1 \mathrm{~h}$ at $-78^{\circ} \mathrm{C}$ and the reaction mixture was brought to room temperature over a period of $1 \mathrm{~h}$ and further stirred for 2-6 h. The reaction was then quenched with $10 \%$ aq. $\mathrm{NH}_{4} \mathrm{Cl}(15 \mathrm{~mL})$ and the resulting solution was concentrated. The residue was diluted with ethyl acetate $(50 \mathrm{~mL})$ and the layers were separated. The aqueous layer was extracted with ethyl acetate $(3 \times 25 \mathrm{~mL})$. The combined extracts were washed with $\mathrm{H}_{2} \mathrm{O}(15 \mathrm{~mL})$, brine $(15 \mathrm{~mL})$, dried (anhydrous. $\mathrm{Na}_{2} \mathrm{SO}_{4}$ ), and concentrated under reduced pressure. The crude product was purified by column chromatography on silica gel or by recrystallization to obtain a pure product.

\section{2-Geranyl-3-methyl naphthoquinone (2) ${ }^{1}$ :}

Naphthalene-2-carboxylic acid methyl ester (43) (370 mg, 1mmol) was treated with LiOBu- $t$ (256 mg, $3.2 \mathrm{mmol}$ ) at $0{ }^{\circ} \mathrm{C}$ in dry THF, after 15 minutes stirring at rt it was then refluxed for $3 \mathrm{~h}$. The reaction was quenched by $3 \mathrm{~N} \mathrm{HCl}$ and was extracted with EtOAc, water and brine and dried over anhydrous $\mathrm{Na}_{2} \mathrm{SO}_{4}$. The crude

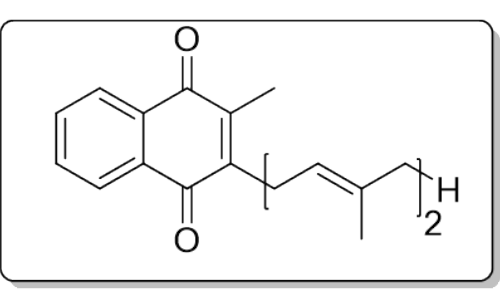


residue was purified by column chromatography which afforded pure compound 2 as yellow oil (123 mg, 40\%) and as a 5:3 mixture of $E$ and $Z$ isomers. ${ }^{1} \mathrm{H}$ NMR (400 $\left.\mathrm{MHz}, \mathrm{CDCl}_{3}\right): 1.56$ (s, 3H), 1.62 (s, 3H), 1.78 (s, 3H), 2.07-1.97 (m, 4H), 2.20 (s, 3H), 3.39 (d, 2H, J = 6.6 Hz), 5.15-5.00 (m, 2H), 7.74-7.71 (m, 2H), 8.10-8.00 (m, 2H). ${ }^{13} \mathrm{C}$ NMR (150 MHz, $\left.\mathrm{CDCl}_{3}\right): \delta$ 11.8, 11.9, 12.6, 12.7, 16.3, 16.5, 17.7, 25.6, 25.7, 26.0, 26.4, 39.7, 116.6, 116.7, 119.1, 119.2, 123.9, 124.0, 124.1, 126..2, 126.3, 127.0, 131.6, 132.0, 132.2, 133.3, 133.4, 137.5, 139.0, 143.4, 146.2, 184.6, 185.5 .

\section{Dimethyl 4-hydroxy-2-methyl-1-oxo-1,2-dihydronaphthalene-2,3-dicarboxylate (23):}

According to the general procedure for annulation , the condensation of $\mathbf{8 e}$ (159 mg, $1 \mathrm{mmol}$ ) with dimethyl 2-methylmaleate 22 (174 mg, $1.1 \mathrm{mmol})$ in the presence of LiOBu-t(256 mg, $3.2 \mathrm{mmol})$ produced compound 23 as yellow oil (214 mg, 74\%). $v_{\max }\left(\mathrm{KBr}, \mathrm{cm}^{-1}\right): 1678,1591,1478$, 1274, 985; ${ }^{1} \mathrm{H}$ NMR (600 MHz, $\left.\mathrm{CDCl}_{3}\right): \delta 12.9$ (s, 1H), $8.12-$ $8.07(\mathrm{~m}, 2 \mathrm{H}), 7.78$ (t, 1H, $J=6.6 \mathrm{~Hz}), 7.63$ (t, 1H, $J=6.6 \mathrm{~Hz})$,

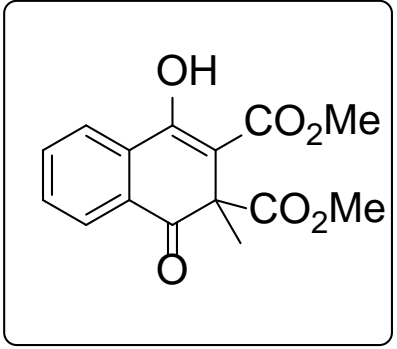
3.85 (s, 3H), 3.69 (s, 3H), 1.64 (s, 3H); ${ }^{13} \mathrm{C}$ NMR (150 MHz, $\mathrm{CDCl}_{3}$ ): $\delta$ 194.6, 171.2, 170.8, 161.6, 135.1, 133.0, 131.8, 129.7, 127.6, 125.2, 102.9, 56.5, 52.8, 52.2, 23.8. HRMS (ES+) $\mathrm{m} / \mathrm{z}$ calcd for $\mathrm{C}_{15} \mathrm{H}_{15} \mathrm{O}_{6}[\mathrm{M}+\mathrm{H}]^{+}$291.0869, found 291.0872.

Methyl 2-(1,4-dioxo-1,4-dihydronaphthalen-2-yl)acetate (25): According to the general procedure for annulation , the condensation of $\mathbf{8 e}(159 \mathrm{mg}, 1$ mmol) with dimethyl itaconate 24 (174 $\mathrm{mg}, 1.1 \mathrm{mmol})$ in the presence of LiOBu-t(256 mg, $3.2 \mathrm{mmol})$ produced compound 25 as yellow solid (130 mg, 57\%). M.p. $102-104^{\circ} \mathrm{C}$. $v_{\max }(\mathrm{KBr}$, $\left.\mathrm{cm}^{-1}\right): 1768,1687,1598,1276,1220,773 ;{ }^{1} \mathrm{H}$ NMR (600 MHz, $\left.\mathrm{CDCl}_{3}\right): \delta 8.13-8.09(\mathrm{~m}, 2 \mathrm{H}), 7.79-7.76(\mathrm{~m}, 2 \mathrm{H}), 6.95$ (s,

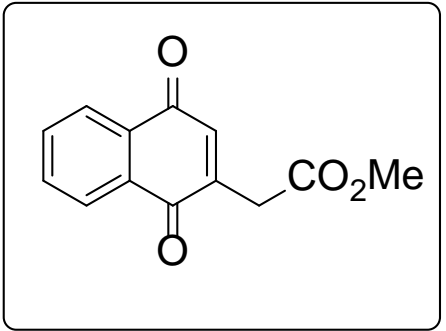
1H), 3.76 (s, 3H), 3.62 (s, 2H); ${ }^{13} \mathrm{C}$ NMR (150 MHz, $\left.\mathrm{CDCl}_{3}\right): \delta 184.6,184.3,169.8,143.9$, 137.3, 133.9, 133.8, 132.2, 131.8, 126.8, 126.3, 52.5, 35.4. HRMS (ES+) $\mathrm{m} / \mathrm{z}$ calcd for $\mathrm{C}_{13} \mathrm{H}_{11} \mathrm{O}_{4}[\mathrm{M}+\mathrm{H}]^{+}$231.0658, found 231.0660.

Methyl (E)-5,9-dimethyl-2-methylenedeca-4,8-dienoate (28c): To a solution of compound 31c (830 mg, $2.5 \mathrm{mmol})$ in dry THF, NaH (198 g, $8.27 \mathrm{mmol})$ was added at $0{ }^{\circ} \mathrm{C}$ and allowed to stir for $30 \mathrm{~min}$. After that paraformaldehyde (2.4 g, $27.56 \mathrm{mmol})$ was added to the reaction mixture and the stirring was continued for $1 \mathrm{~h}$ more at

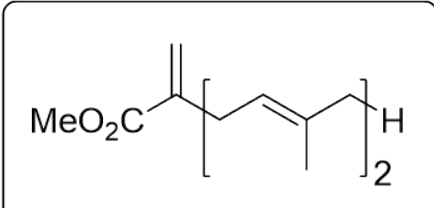


the same temperature. The reaction was monitored by TLC, and after completion of the reaction, it was quenched by the addition of saturated ammonium chloride solution and was extracted with $\mathrm{Et}_{2} \mathrm{O}$, water and brine and dried over anhydrous $\mathrm{Na}_{2} \mathrm{SO}_{4}$. The crude residue was purified by column chromatography which afforded pure compound 28c as colorless oil (378 mg, 68\%). $v_{\max }\left(\mathrm{KBr}, \mathrm{cm}^{-1}\right): 1725,1680,1453,1378,1272,1210,1192,1054,780 .{ }^{1} \mathrm{H}$ NMR (600 MHz, $\left.\mathrm{CDCl}_{3}\right): \delta 6.12(\mathrm{~s}, 1 \mathrm{H}), 5.52(\mathrm{~s}, 1 \mathrm{H}), 5.18-5.07$ (m, 2H), $3.74(\mathrm{~s}, 3 \mathrm{H}), 2.98$ (d, $2 \mathrm{H}, J=7.2 \mathrm{~Hz}$ ), $2.10-2.02$ (m, 4H), 1.67 (s, 3H), 1.61 (s, 3H), 1.58 (s, 3H); ${ }^{13} \mathrm{C}$ NMR (150 MHz, $\left.\mathrm{CDCl}_{3}\right): \delta 167.7,139.5,137.7,124.4,124.2,120.4,120.3,51.7,39.7,30.1,26.5$, 25.6, 17.6, 15.8. HRMS (ES+) $\mathrm{m} / z$ calcd for $\mathrm{C}_{14} \mathrm{H}_{23} \mathrm{O}_{2}[\mathrm{M}+\mathrm{H}]^{+}$223.1699, found 223.1701.

\section{2-Ethylidene-5-methyl-hex-4-enoic acid methyl ester (28d)}

To a solution of compound $31 \mathrm{~b}(1.4 \mathrm{~g}, 5.03 \mathrm{mmol})$ in dry THF, NaH (145 mg, $6.04 \mathrm{mmol}$ ) was added at $0{ }^{\circ} \mathrm{C}$ and allowed to stir for $30 \mathrm{~min}$. After that acetaldehyde (664 mg, $15.0 \mathrm{mmol}$ ) was added to the reaction mixture and the stirring was continued for $1 \mathrm{~h}$ more at the same temperature. The reaction was monitored by TLC, and after completion

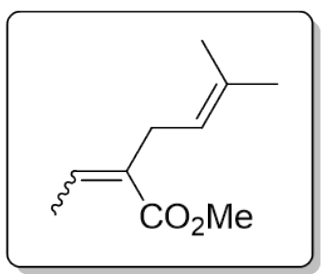
of the reaction, it was quenched by the addition of saturated ammonium chloride solution and was extracted with $\mathrm{Et}_{2} \mathrm{O}$, water and brine and dried over anhydrous $\mathrm{Na}_{2} \mathrm{SO}_{4}$. The crude residue was purified by column chromatography which afforded pure compound 28d (mixture of $\boldsymbol{E}+\boldsymbol{Z}$ isomer) as colorless oil (1.17g, $72 \%) \cdot v_{\max }\left(\mathrm{KBr}, \mathrm{cm}^{-1}\right): 2923,2853$, 1734,1458, 1376, 1219. ${ }^{1} \mathrm{H}$ NMR (600 MHz, $\left.\mathrm{CDCl}_{3}\right): \delta 6.84(\mathrm{q}, 1 \mathrm{H}, J=7.2 \mathrm{~Hz}, E), 5.99$ (q,0.3H, $J=7.2 \mathrm{~Hz}, Z$ ), 5.12-5.01 (m, 1.3H, E+Z), 3.75 (s, .9H, Z), 3.73 (s, 3H, E), 3.02 (d, $J$ $=6.6 \mathrm{~Hz}, 2 \mathrm{H}, E), 2.93$ (d, $J=6.6 \mathrm{~Hz}, 0.6 \mathrm{H}, Z), 1.96$ (d, 0.9H, $J=7.2 \mathrm{~Hz}, Z$ ), 1.82 (d,3H, $J=$ 7.2 Hz, E), 1.73-1.62 (m, 8H). ${ }^{13} \mathrm{C}$ NMR (150 MHz, $\left.\mathrm{CDCl}_{3}\right): \delta 168.6,168.3,137.2,136.2$, 133.5, 132.5, 132.1, 131.7, 121.5, 121.4, 51.6, 51.1, 32.6, 25.7, 25.7, 25.6, 17.8, 17.6, 15.6, 14.2. HRMS (ES+) $m / z$ calcd for $\mathrm{C}_{10} \mathrm{H}_{17} \mathrm{O}_{2}[\mathrm{M}+\mathrm{H}]^{+}$169.1229, found 169.1232.

\section{2-Ethylidene-pent-4-enoic acid methyl ester (28e) ${ }^{2}$ :}

To a solution of compound 31a (2.0 g, $8.0 \mathrm{mmol})$ in dry THF, NaH (257 mg, $11.7 \mathrm{mmol})$ was added at $0{ }^{\circ} \mathrm{C}$ and allowed to stir for $30 \mathrm{~min}$. After that acetaldehyde (1.76 g, $40.0 \mathrm{mmol}$ ) was added to the reaction mixture and the stirring was continued for $1 \mathrm{~h}$ more at the same temperature. The reaction was monitored by TLC, and after completion of the reaction, it was

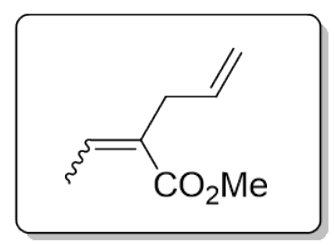
quenched by the addition of saturated ammonium chloride solution and was extracted with 
$\mathrm{Et}_{2} \mathrm{O}$, water and brine and dried over anhydrous $\mathrm{Na}_{2} \mathrm{SO}_{4}$. The crude residue was purified by column chromatography which afforded pure compound 28e (mixture of $\boldsymbol{E}+\boldsymbol{Z}$ isomer) as colorless oil (1.72g, 65\%). $v_{\max }\left(\mathrm{KBr}, \mathrm{cm}^{-1}\right)$ : 2930, 1728,1437, 1320, 1222, 658. ${ }^{1} \mathrm{H}$ NMR (600 MHz, $\left.\mathrm{CDCl}_{3}\right): \delta 6.96(\mathrm{q}, 1 \mathrm{H}, J=7.2 \mathrm{~Hz}, E), 6.05$ (q, 0.4H, $J=7.2 \mathrm{~Hz}, Z$ ), 5.85-5.79 (m, 1.4H, E+Z), 5.07-4.98 (m, 2.8H, E+Z), 3.75 (s, 1.2H, Z), 3.74 (s, $3 \mathrm{H}, E$ ), 3.09 (d, $J=6.0 \mathrm{~Hz}$, 2H, E), 3.01 (d, 0.8H, $J=6.0 \mathrm{~Hz}, Z$ ), 2.00 (d, 1.2H, $J=7.2 \mathrm{~Hz}, Z$ ), 1,81 (d, 3H, $J=6.6 \mathrm{~Hz}$, E). ${ }^{13} \mathrm{C}$ NMR (150 MHz, $\left.\mathrm{CDCl}_{3}\right): \delta 168.2,168.0,138.8,137.9,136.1,135.1,130.9,130.6$, 116.0, 115.0, 51.7, 51.2, 38.3, 30.4, 15.7, 14.2. HRMS (ES+) $m / z$ calcd for $\mathrm{C}_{8} \mathrm{H}_{13} \mathrm{O}_{2}[\mathrm{M}+$ $\mathrm{H}]^{+}$141.0916, found 141.0916 .

2-Ethylidene-5,9-dimethyl-deca-4,8-dienoic acid methyl ester (28f): To a solution of compound 31c (1.66 g, $4.79 \mathrm{mmol}$ ) in dry THF, NaH (400 mg, $16.54 \mathrm{mmol})$ was added at 0 ${ }^{\circ} \mathrm{C}$ and allowed to stir for $30 \mathrm{~min}$. After that acetaldehyde (1.1g, $\left.25.0 \mathrm{mmol}\right)$ was added to the reaction mixture and the stirring was continued for $1 \mathrm{~h}$ more at the same temperature. The reaction was monitored by TLC, and after completion of the reaction, it was quenched by the addition of saturated ammonium chloride solution and was extracted with $\mathrm{Et}_{2} \mathrm{O}$, water and brine and dried over anhydrous $\mathrm{Na}_{2} \mathrm{SO}_{4}$. The crude

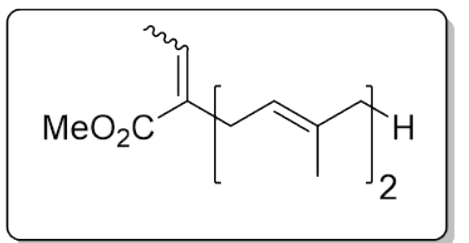
residue was purified by column chromatography which afforded pure compound $\mathbf{2 8 f}$ (mixture of $\boldsymbol{E}+\boldsymbol{Z}$ isomer) as colorless oil (1.25g, 52\%). $v_{\max }\left(\mathrm{KBr}, \mathrm{cm}^{-1}\right): 2954,2925,2854,1719$, 1685, 1458, 1377, 1275, 1219, 1192, 1125, 1054, 772. ${ }^{1} \mathrm{H}$ NMR (600 MHz, $\left.\mathrm{CDCl}_{3}\right): \delta 6.85$ (q, $J=7.2 \mathrm{~Hz}, 1 \mathrm{H}, E), 5.99$ (q, $0.3 \mathrm{H}, J=6.6 \mathrm{~Hz}, \mathrm{Z}), 5.14-4.65$ (m, 3H, E+Z), 3.75 (s, 1H, Z), 3.73 (s, 3H, E), 3.033 (d, 2H, $J=7.2 \mathrm{~Hz}, E$ ), 2.95 (d, $0.6 \mathrm{H}, J=6.6 \mathrm{~Hz}, Z$ ), 2.10-1.94 (m, 6.6H, E+Z), 1.82 (d, 3H, $J=7.2 \mathrm{~Hz}, E), 1.70-1.52(\mathrm{~m}, 12.2 \mathrm{H}, E+Z) .{ }^{13} \mathrm{C}$ NMR $(150 \mathrm{MHz}$, $\left.\mathrm{CDCl}_{3}\right): \delta 168.3,168.3,137.3,136.1,135.7,132.6,131.3,124.2,121.6,121.4,121.2,109.7$, 51.6, 51.1, 39.7, 39.7, 39.2, 37.3, 32.4, 26.6, 26.6, 25.8, 25.7, 25.5,22.4, 17.7, 17.7, 16.1, 16.0, 15.9, 15.7.14.2. IR ( $\left.\mathrm{KBr}, \mathrm{cm}^{-1}\right)$ : HRMS (ES+) $\mathrm{m} / \mathrm{z}$ calcd for $\mathrm{C}_{15} \mathrm{H}_{25} \mathrm{O}_{2}[\mathrm{M}+\mathrm{H}]^{+}$ 237.1855, found 237.1859.

Methyl 2-(diethoxyphosphoryl)pent-4-enoate (31a): To a solution of methyl-2-diethoxyphosphorylacetate (30) (5 g, 23.8 mmol) in dry DMF $(20 \mathrm{~mL})$ at $0{ }^{\circ} \mathrm{C}$, solid $\mathrm{KOBu}-t(3.5 \mathrm{~g}, 31.3$ mmol) was added and the resulting mixture was stirred for $15 \mathrm{~min}$.

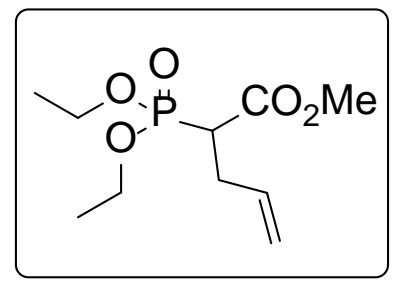
Then allyl bromide (29a) $(1.6 \mathrm{~mL}, 19.1 \mathrm{mmol})$ was added to the reaction mixture and the reaction was allowed to stir for $20 \mathrm{~h}$ more in rt. After that the reaction was quenched by 
addition of $15 \mathrm{~mL} 3 \mathrm{~N} \mathrm{HCl}$. The aqueous layer was extracted with diethyl ether, and the combined organic layer was washed with brine, dried with $\mathrm{Na}_{2} \mathrm{SO}_{4}$ and concentrated under reduced pressure. The initial product was purified by flash chromatography to afford compound 31a as a colorless oil (3.7 g, 80\%). $v_{\max }\left(\mathrm{KBr}, \mathrm{cm}^{-1}\right)$ : 2932, 1720, 1672, 1444, 1395, 1355, 1320, 1249, 750. ${ }^{1} \mathrm{H}$ NMR (600 MHz, $\left.\mathrm{CDCl}_{3}\right): \delta 5.78-5.65$ (m, 2H), $5.05-$ 4.96 (m, 2H), 4.09 - 4.04 (m, 3H), 3.66 (s, 2H), 3.01 - 2.50 (m, 2H), 2.61- 2.38 (m, 4H), 1.26 - 1.23 (m, 4H). ${ }^{13} \mathrm{C}$ NMR (150 MHz, $\left.\mathrm{CDCl}_{3}\right): \delta 169.1,169.0,134.5,134.4,133.1,133.0$, 118.5, 117.1, 62.8, 62.8, 62.7, 62.6, 52.3, 52.2, 45.6, 44.8, 36.3, 30.9, 16.3, 16.2. HRMS (ES+) $\mathrm{m} / \mathrm{z}$ calcd for $\mathrm{C}_{10} \mathrm{H}_{20} \mathrm{O}_{5} \mathrm{P}[\mathrm{M}+\mathrm{H}]^{+}$251.1049, found 251.1052 .

Methyl-2-(diethoxyphosphoryl)-5-methylhex-4-enoate (31b) ${ }^{3}$ : To a solution of methyl-2diethoxyphosphorylacetate (30) (5 g, $23.8 \mathrm{mmol}$ ) in dry DMF $(20 \mathrm{~mL})$ at $0{ }^{\circ} \mathrm{C}$, solid $\mathrm{KBuO}-\mathrm{t}(3.5 \mathrm{~g}, 31.3 \mathrm{mmol})$ was added and the resulting mixture was stirred for $15 \mathrm{~min}$. Then prenylbromide (29b) $(2.2 \mathrm{~mL}, 19.1 \mathrm{mmol})$ was added to the reaction mixture and the reaction was allowed to stir for $20 \mathrm{~h}$

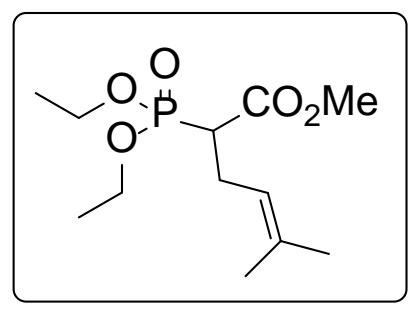
more in rt. After that the reaction was quenched by addition of $15 \mathrm{~mL} 3 \mathrm{~N} \mathrm{HCl}$. The aqueous layer was extracted with diethyl ether, and the combined organic layer was washed with brine, dried with $\mathrm{Na}_{2} \mathrm{SO}_{4}$ and concentrated under reduced pressure. The initial product was purified by flash chromatography to afford compound 31b as a colorless oil (5.56 g, 70\%). $v_{\max }\left(\mathrm{KBr}, \mathrm{cm}^{-1}\right): 2929,1735,1675,1438,1388,1376,1330,1246,1163,850 .{ }^{1} \mathrm{H}$ NMR $(600$ $\mathrm{MHz}, \mathrm{CDCl}_{3}$ ): $\delta 5.03(\mathrm{t}, 1 \mathrm{H}, J=3.4 \mathrm{~Hz}), 4.14(\mathrm{~m}, 4 \mathrm{H}), 3.73(\mathrm{~s}, 3 \mathrm{H}), 2.95(\mathrm{~m}, 1 \mathrm{H}), 2.61(\mathrm{~m}$, 2H), 1.70 (d, 3H, $J=13.4 \mathrm{~Hz}$ ), 1.64 (d, 3H, $J=4.4 \mathrm{~Hz}), 1.33$ (d, 6H, $J=3.6 \mathrm{~Hz}) ;{ }^{13} \mathrm{C} \mathrm{NMR}$ (150 MHz, $\left.\mathrm{CDCl}_{3}\right): \delta 169.6,134.9,120.5,62.7,62.6,52.5,47.4,44.8,25.9,17.8,16.6,16.4$. HRMS (ES+) $m / z$ calcd for $\mathrm{C}_{12} \mathrm{H}_{24} \mathrm{O}_{5} \mathrm{P}[\mathrm{M}+\mathrm{H}]^{+}$279.1362, found 279.1369.

Methyl (E)-2-(diethoxyphosphoryl)-5,8-dimethylnona-4,7-dienoate (31c): Compound 31c was synthesized from geranyl bromide $29 c(3.79 \mathrm{~mL}, 19.1$ mmol)and methyl-2-diethoxyphosphorylacetate (30) (5 g, $23.8 \mathrm{mmol}$ ) following the same procedure described above. Column chromatography of the crude residue afforded 31c

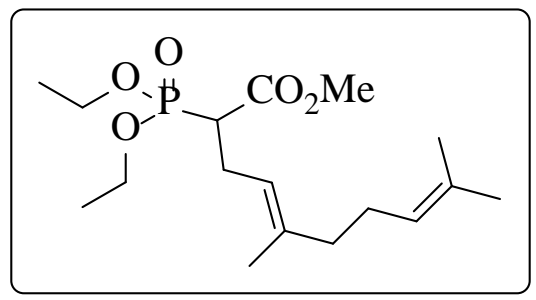
as colorless liquid with characteristic smell $(6.1 \mathrm{~g}, 75 \%) . v_{\max }\left(\mathrm{KBr}, \mathrm{cm}^{-1}\right): 1738,1672,1435$, 1390, 1372, 1332, 1246, 1160, 865. ${ }^{1} \mathrm{H}$ NMR (600 MHz, $\left.\mathrm{CDCl}_{3}\right): \delta 5.07$ (t, 2H, $J=7.2 \mathrm{~Hz}$ ), 
4.21 - 4.13 (m, 4H), 3.75 (s, 3H), $3.01-2.52$ (m, 3H), 2.06 - 1.97 (m, 4H), 1.69 (s, 3H), 1.65 (s, 3H), 1.60 (s, 3H), $1.37-1.28$ (m, 6H). $\left.{ }^{13} \mathrm{C} \mathrm{NMR} \mathrm{(150} \mathrm{MHz,} \mathrm{CDCl}_{3}\right): \delta 169.5,138.4$, 131.5, 123.9, 120.3, 120.2, 62.8, 62.7, 62.6, 62.6, 52.3, 46.5, 45.6, 39.6, 26.5, 25.7, 25.6, 17.7, 16.4, 16.3, 16.3, 16.0. HRMS (ES+) $\mathrm{m} / \mathrm{z}$ calcd for $\mathrm{C}_{17} \mathrm{H}_{32} \mathrm{O}_{5} \mathrm{P}[\mathrm{M}+\mathrm{H}]^{+}$347.1988, found 347.1991.

Methyl 1-hydroxy-4-((3-methylbut-2-en-1-yl)oxy)-2-naphthoate (32): According to the general procedure for annulation , the condensation of $\mathbf{8 e}$ (159 $\mathrm{mg}, 1 \mathrm{mmol})$ with $\alpha$-prenyl acrylate $28 \mathbf{b}(170 \mathrm{mg}, 1.1 \mathrm{mmol})$ in the presence of LiOBu-t(256 mg, $3.2 \mathrm{mmol})$ produced compound 32 as white gum $(237 \mathrm{mg}, 83 \%) . v_{\max }\left(\mathrm{KBr}, \mathrm{cm}^{-1}\right): 1683,1617$, 1353, 1076, 775; ${ }^{1} \mathrm{H}$ NMR (600 MHz, $\mathrm{CDCl}_{3}$ ): 11.63 (s, 1H), 8.41 (d, $1 \mathrm{H}, J=8.4 \mathrm{~Hz}), 8.25$ (d, 1H, $J=8.4 \mathrm{~Hz}), 7.65-7.51$ (m, 2H),

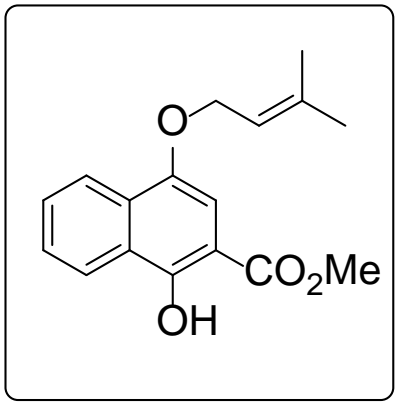
7.07 (s, 1H), $5.64-5.62$ (m, 1H), 4.67 (d, 2H, $J=6.6$ Hz), 4.02 (s, 3H), 1.86 (s, 3H), 1.82 (s, 3H); ${ }^{13} \mathrm{C}$ NMR (50 MHz, $\left.\mathrm{CDCl}_{3}\right): \delta 171.5,155.7,147.0,137.9,130.4,129.1,126.5,125.7$, 123.9, 122.3, 120.0, 104.4, 101.9, 65.6, 52.4, 26.0, 18.4. HRMS (ES+) m/z calcd for $\mathrm{C}_{17} \mathrm{H}_{19} \mathrm{O}_{4}[\mathrm{M}+\mathrm{H}]^{+}$287.1284, found 287.1287.

2-Allylnaphthalene-1,4-dione (33) ${ }^{4}$ : According to the general procedure for annulation, the condensation of $\mathbf{8 e}(159 \mathrm{mg}, 1 \mathrm{mmol})$ with $\alpha$-allyl acrylate $28 \mathrm{a}$ (140 $\mathrm{mg}, 1.1 \mathrm{mmol})$ in the presence of LiOBu- $t$ (256 mg, $3.2 \mathrm{mmol}$ ) produced compound $\mathbf{9 0}$ as orange solid (128 mg, 65\%). M. p. $126-128^{\circ} \mathrm{C} . v_{\max }\left(\mathrm{KBr}, \mathrm{cm}^{-}\right.$ $\left.{ }^{1}\right): 1637,1484,1218,1058,771 ;{ }^{1} \mathrm{H}$ NMR $\left(600 \mathrm{MHz}, \mathrm{CDCl}_{3}\right): \delta$ $8.13-8.08$ (m, 2H), 7.50 (t, 2H, $J=2.4 \mathrm{~Hz}$ ), 6.83 (s, 1H), $5.95-$ 5.88 (m, 1H), 5.25 - 5.23 (m, 2H), $3.36-3.35$ (m, 2H); ${ }^{13} \mathrm{C}$ NMR

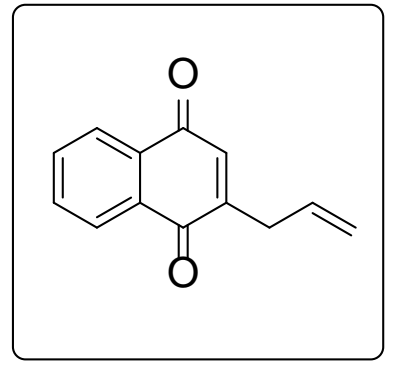
$\left(150 \mathrm{MHz}, \mathrm{CDCl}_{3}\right): \delta 185.1,184.9,149.9,135.2,133.8,133.7,133.0,132.2,132.1,126.6$, 126.1, 118.9, 33.5.

\section{Methyl (E)-4-((3,7-dimethylocta-2,6-dien-1-yl)oxy)-1-hydroxy-2-naphthoate (34a):}

According to the general procedure for annulation, the condensation of $\mathbf{8 e}$ (159 mg, $1 \mathrm{mmol}$ ) with $\alpha$-geranyl acrylate 28c (140 mg, $1.1 \mathrm{mmol})$ in the presence of LiOBu-t(256 mg, $3.2 \mathrm{mmol})$ produced compound $\mathbf{3 4 a}$ as white oil (177 mg, 50\%). $v_{\max }\left(\mathrm{KBr}, \mathrm{cm}^{-1}\right): 1687,1620,1355$, 1079, 780. ${ }^{1} \mathrm{H}$ NMR (600 MHz, $\left.\mathrm{CDCl}_{3}\right): \delta 11.63(\mathrm{~s}, 1 \mathrm{H}), 8.41$ (d, $1 \mathrm{H}, J=8.4 \mathrm{~Hz}), 8.26$ (d, 1H, $J=8.4 \mathrm{~Hz}), 7.66-7.57$ (m,

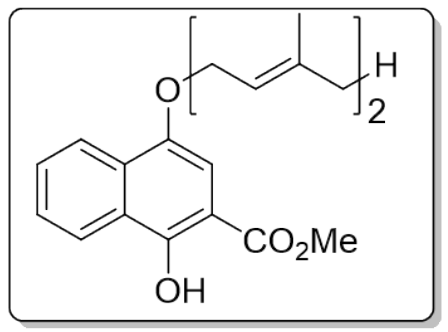


2H), 7.07 (s, 1H), 5.63 (t, 1H, $J=6 \mathrm{~Hz}), 5.16$ (t, 1H, $J=6.6 \mathrm{~Hz}), 4.69$ (d, 2H, $J=6.6 \mathrm{~Hz}$ ), 4.01 (s, 3H), 1.87-1.81 (m, 4H), 1.73 (s, 3H), 1.71 (s, 3H), 1.65 (s, 3H); ${ }^{13} \mathrm{C}$ NMR (150 MHz, $\left.\mathrm{CDCl}_{3}\right): \delta 171.5,155.7,147.1,141.2,131.9,130.4,129.1,126.5,125.7,124.0,123.9,122.3$, 119.9, 104.5, 102.1, 65.7, 52.4, 39.8, 26.6, 25.9, 17.9, 16.9. HRMS (ES+) m/z calcd for $\mathrm{C}_{22} \mathrm{H}_{27} \mathrm{O}_{4}[\mathrm{M}+\mathrm{H}]^{+}$355.1910, found 355.1914.

(E)-2-(3,7-Dimethylocta-2,6-dien-1-yl)naphthalene-1,4-dione (34b) ${ }^{5}$ : This compound was obtained as a co product of compound 34a. White viscous material (117 mg, 40\%). $v_{\max }$ $\left(\mathrm{KBr}, \mathrm{cm}^{-1}\right): 1664,1637,1590,1359,1255,721 ;{ }^{1} \mathrm{H}$ NMR $\left(600 \mathrm{MHz}, \mathrm{CDCl}_{3}\right): \delta 8.12-8.06(\mathrm{~m}, 2 \mathrm{H}), 7.74-7.72(\mathrm{~m}$, 2H), 6.77 (s, 1H), 5.25 (t, 1H, $J=7.2 \mathrm{~Hz}$ ), 5.11 (t, 1H, $J=6.6$ $\mathrm{Hz}$ ), 3.30 (d, 2H, $J=7.2 \mathrm{~Hz}$ ), $2.15-2.10$ (m, 4H), 1.71 (s,

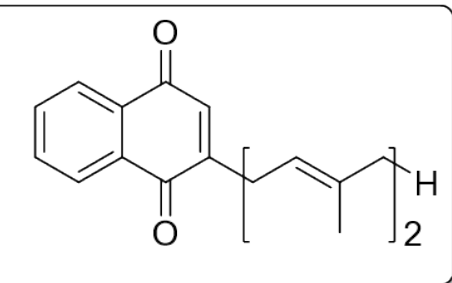
3H), 1.68 (s, 3H), 1.63 (s, 3H); ${ }^{13} \mathrm{C}$ NMR (150 MHz, $\left.\mathrm{CDCl}_{3}\right): \delta 185.4,185.3,150.8,140.0$, 134.6, 133.6, 133.5, 132.3, 132.2, 131.8, 126.5, 126.1, 123.9, 118.1, 39.7, 27.9, 26.4, 25.7, 17.7, 16.1 .

\section{2-Allyl-3-methyl-1,4-dioxo-1,2,3,4-tetrahydro-naphthalene-2-carboxylic acid methyl ester (39):}

According to the general procedure for annulation, the condensation of $\mathbf{8 e}(159 \mathrm{mg}, 1 \mathrm{mmol})$ with $\alpha$-allyl acrylate $\mathbf{2 8 e}$ (167 $\mathrm{mg}, 1.2 \mathrm{mmol})$ in the presence of LiOBu-t (256 mg, $3.2 \mathrm{mmol})$ produced compound 39 as colorless oil (332 mg, 82\%). $v_{\max }(\mathrm{KBr}$, $\left.\mathrm{cm}^{-1}\right):$ 2934, 1714, 1423, 1246, 953, 771. ${ }^{1} \mathrm{H}$ NMR $(600 \mathrm{MHz}$,

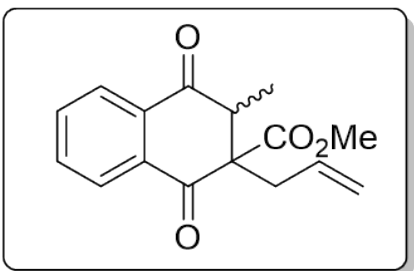
$\mathrm{CDCl}_{3}$ ): $\delta 1.19$ (d, 6H, $J=7.2 \mathrm{~Hz}$ ), 1.31 (d, 3H, $J=6.6 \mathrm{~Hz}$ ), 2.53 (dd, $1 \mathrm{H}, J=9.6 \mathrm{~Hz}$ ), 2.722.75 (m, 2H), 2.79-2.83 (m, 2H), 3.19 (q, 1H, $J=6.6 \mathrm{~Hz}$ ), 3.56 (s, 3H), 3.59 (q, 2H, $J=7.2$ Hz), 3.65 (s, 6H), 5.00-5.06 (m, 4H), 5.13 (d, 1H, $J=10.2$ Hz), 5.23 (d, 1H, $J=16.8$ ), 5.635.68 (m, 3H), 7.70-7.77 (m, 6H), 7.98 (d, 2H, $J=7,2 \mathrm{~Hz}), 8.06$ (d, 4H, $J=7.2 \mathrm{~Hz}) .{ }^{13} \mathrm{C}$ NMR (150 MHz, $\left.\mathrm{CDCl}_{3}\right): \delta 197.1,196.4,193.8,192.1,170.6,169.1,136.1,134.7,134.6,134.3$, 134.0, 133.7, 133.5, 133.4, 132.5, 131.6, 127.6, 127.1, 127.0, 126.2, 120.1, 119.5, 65.4, 64.8, 53.0, 53.0, 47.9, 46.5, 35.1, 34.9, 12.4, 9.4. HRMS (ES+) $\mathrm{m} / \mathrm{z}$ calcd for $\mathrm{C}_{16} \mathrm{H}_{17} \mathrm{O}_{4}[\mathrm{M}+\mathrm{H}]^{+}$ 273.1128, found 273.1127 . 


\section{2-Allyl-3-methyl naphthoquinone (40) ${ }^{6}$ :}

Naphthalene-2-carboxylic acid methyl ester (39) (272 mg, $1 \mathrm{mmol})$ was treated with LiOBu-t $(256 \mathrm{mg}, 3.2 \mathrm{mmol})$ at $0{ }^{\circ} \mathrm{C}$ in dry THF, after 15 minutes stirring at rt it was then refluxed for 3 h. The reaction was quenched by $3 \mathrm{~N} \mathrm{HCl}$ and was extracted with

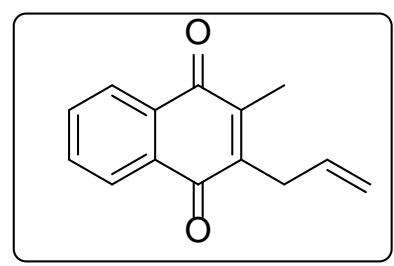
EtOAc, water and brine and dried over anhydrous $\mathrm{Na}_{2} \mathrm{SO}_{4}$. The crude residue was purified by column chromatography which afforded pure compound $\mathbf{4 0}$ as yellow oil (96 mg, 45\%). ${ }^{1} \mathrm{H}$ NMR (400 MHz, $\left.\mathrm{CDCl}_{3}\right)$ : $\delta$ 8.10-8.07 (m, 2H), 7.74-7.68 (m, 2H), 5.89-5.79 (m, 2H), 5.095.06 (m, 2H), 3.43 (d, 2H, $J=6.4 \mathrm{~Hz}), 2.20$ (s, 3H).

\section{3-Methyl-2-(3-methyl-but-2-enyl)-1,4-dioxo-1,2,3,4-tetrahydro-naphthalene-2- carboxylic acid methyl ester (41)}

According to the general procedure for annulation , the condensation of $\mathbf{8 e}$ (100 $\mathrm{mg}, 0.63$ mmol) with $\alpha$-allyl acrylate $\mathbf{2 8 d}$ (120 mg, $1.1 \mathrm{mmol})$ in the presence of LiOBu-t (161 mg, 3.2 mmol) produced compound $\mathbf{4 1}$ as colourless oil (259 mg, 73\%). $v_{\max }\left(\mathrm{KBr}, \mathrm{cm}^{-1}\right): 2945,1734,1423,1257,769,657 .{ }^{1} \mathrm{H}$ NMR $(600$ $\left.\mathrm{MHz}, \mathrm{CDCl}_{3}\right): \delta 8.11-8.00(\mathrm{~m}, 3 \mathrm{H}), 8.01$ (d, $\left.1 \mathrm{H}, J=7.2 \mathrm{~Hz}\right), 7.78-$ 7.72 ( m, 4H), 4.95-4.93 (m, 2H), 3.68 (s, 3H), 3.63 (q, 1H, $J=7.2$ Hz), 3.58 (s, 3H), 3.35-3.33 (m, 1H), $3.23(\mathrm{q}, 1 \mathrm{H}, J=6.0 \mathrm{~Hz})$,

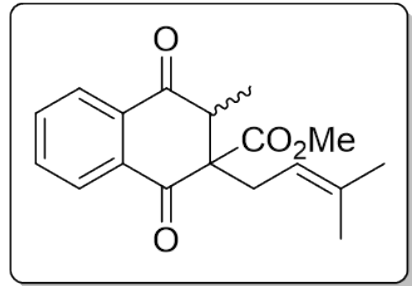
2.82-2.71 (m, 2H), 2.67-2.63 (m, 1H), 1.73 (s, 3H), 1.67 (s, 3H), 1.58 (s, 3H), 1.44 (s, 3H), 1.31 (s, 3H), 1.28 (d, 3H, $J=7.2 \mathrm{~Hz}), 1.21$ (d, 3H, $J=7.2 \mathrm{~Hz}) .{ }^{13} \mathrm{C}$ NMR (150 MHz, $\left.\mathrm{CDCl}_{3}\right)$ : $\delta 197.2,196.8,194.7,192.6,171.0,169.5,136.6,136.4,136.1,134.6,134.5,134.5,134.1$, 133.9, 133.7, 133.6, 127.6, 127.0, 126.8, 126.2, 118.0, 117.0, 65.7, 68.3, 52.9, 47.9, 46.7, 29.8, 29.3, 26.1, 25.8, 18.2, 17.7, 12.3, 9.9. HRMS (ES+) $\mathrm{m} / \mathrm{z}$ calcd for $\mathrm{C}_{18} \mathrm{H}_{21} \mathrm{O}_{4}[\mathrm{M}+\mathrm{H}]^{+}$ 301.1441, found 301.1443.

2-Prenyl-3-methyl naphthoquinone (42) ${ }^{7}$ : Naphthalene-2carboxylic acid methyl ester (41) (300 mg, $1 \mathrm{mmol}$ ) was treated with LiOBu- $t$ (256 mg, $3.2 \mathrm{mmol}$ ) at $0{ }^{\circ} \mathrm{C}$ in dry THF, after 15 minutes stirring at $\mathrm{rt}$ it was then refluxed for $3 \mathrm{~h}$. The reaction was quenched by $3 \mathrm{~N} \mathrm{HCl}$ and was extracted with EtOAc, water

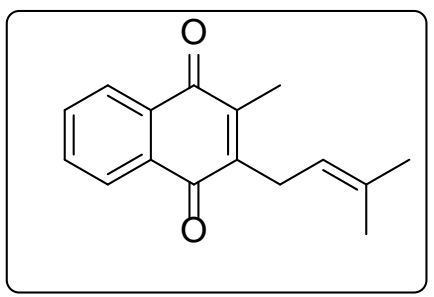
and brine and dried over anhydrous $\mathrm{Na}_{2} \mathrm{SO}_{4}$. The crude residue was purified by column 
chromatography which afforded pure compound 42 as yellow oil (132 mg, 55\%). ${ }^{1} \mathrm{H}$ NMR (400 MHz, $\mathrm{CDCl}_{3}$ ): 8.10 (d, 2H, $J=4.2 \mathrm{~Hz}$ ), 7.73 (d, 2H, $J=4.2 \mathrm{~Hz}$ ), 5.04 (t, 1H, $J=4 \mathrm{~Hz}$ ), 3.38 (d, 2H, $J=7.2 \mathrm{~Hz}$ ), 2.22 (s, 3H), 1.82 (s, 3H), 1.71 (s, 3H).

\section{2-(3,7-Dimethyl-octa-2,6-dienyl)-3-methyl-1,4-dioxo-1,2,3,4-tetrahydro-naphthalene-2-}

carboxylic acid methyl ester (43):According to the general procedure for annulation , the condensation of $\mathbf{8 e}$ (159 mg, $1 \mathrm{mmol}$ ) with $\alpha$-allyl acrylate $\mathbf{2 8 f}$ (266.8 $\mathrm{mg}, 1.2 \mathrm{mmol}$ ) in the presence of LiOBu- $t$ (256 mg, $3.2 \mathrm{mmol}$ ) produced compound 43 as colorless oil $(259.2 \mathrm{mg}, 65 \%) . v_{\max }\left(\mathrm{KBr}, \mathrm{cm}^{-1}\right)$ : 2925, 2367, 1718, 1734, 1700, 1438, 1251, 1220, 957, 772. ${ }^{1} \mathrm{H}$ NMR (600 MHz, $\left.\mathrm{CDCl}_{3}\right): \delta$ 8.01-7.98 (m, 3H), 7.75-7.71 (m, 3H), 4.98-4.58 (m, 4H), 3.66 (s, 3H), 3.62 (q, $J=7.2 \mathrm{~Hz}, 1 \mathrm{H})$,

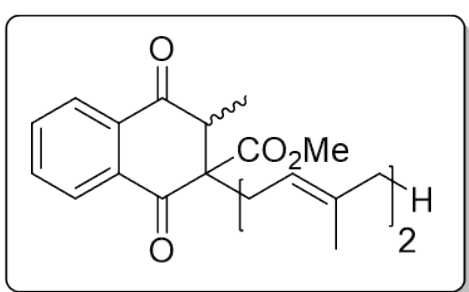

3.56 (s, 2H), 3.40-3.21 (m, 1H), 2.85-2.45 (m, 3H), 2.01-1.97 (m, 2H), 1.90-1.85 (m, 4H), 1.70-1.66 (m, 6H), 1.56 (s, 3H), 1.49 (d, 3H, $J=10.2 \mathrm{~Hz}$ ), 1.41 (s, 3H), 1.26 (d, 2H, $J=6.6$ $\mathrm{Hz}), 1.19$ (d, 3H, $J=7.2 \mathrm{~Hz}) .{ }^{13} \mathrm{C}$ NMR (150 MHz, $\left.\mathrm{CDCl}_{3}\right): \delta 197.6,196.9,194.7,192.6$, 171.0, 169.5, 140.0, 139.7, 136.0, 134.6, 134.5, 134.1, 134.1, 134.0, 133.6, 131.5, 127.6, 127.0, 126.7, 126.2, 123.9, 123.9, 118.2, 117.0, 116.9, 109.9, 65.7, 65.2, 52.9, 47.9, 47.8, 46.7, 39.8, 39.7, 39.3, 37.2, 29.7, 29.3, 29.3, 29.3, 26.2, 26.1, 25.6, 25.5, 25.5, 22.3, 17.7, 17.6, 16.4, 16.0, 15.9, 12.2, 9.8. HRMS (ES+) $\mathrm{m} / \mathrm{z}$ calcd for $\mathrm{C}_{23} \mathrm{H}_{29} \mathrm{O}_{4}[\mathrm{M}+\mathrm{H}]^{+}$369.2067, found

369.2081. 
${ }^{1} \mathrm{H}$ NMR of compound $2\left(\mathrm{CDCl}_{3}, 600 \mathrm{MHz}\right)$

$\mathrm{dm}$

/sj / 440pi - 1h - f688
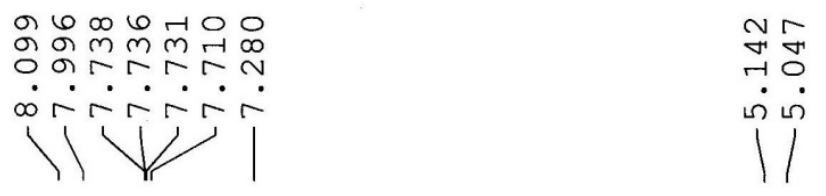

م⿱

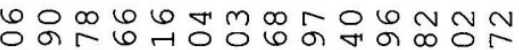

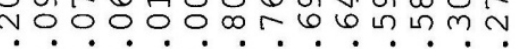

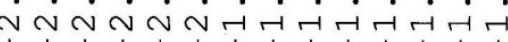

1
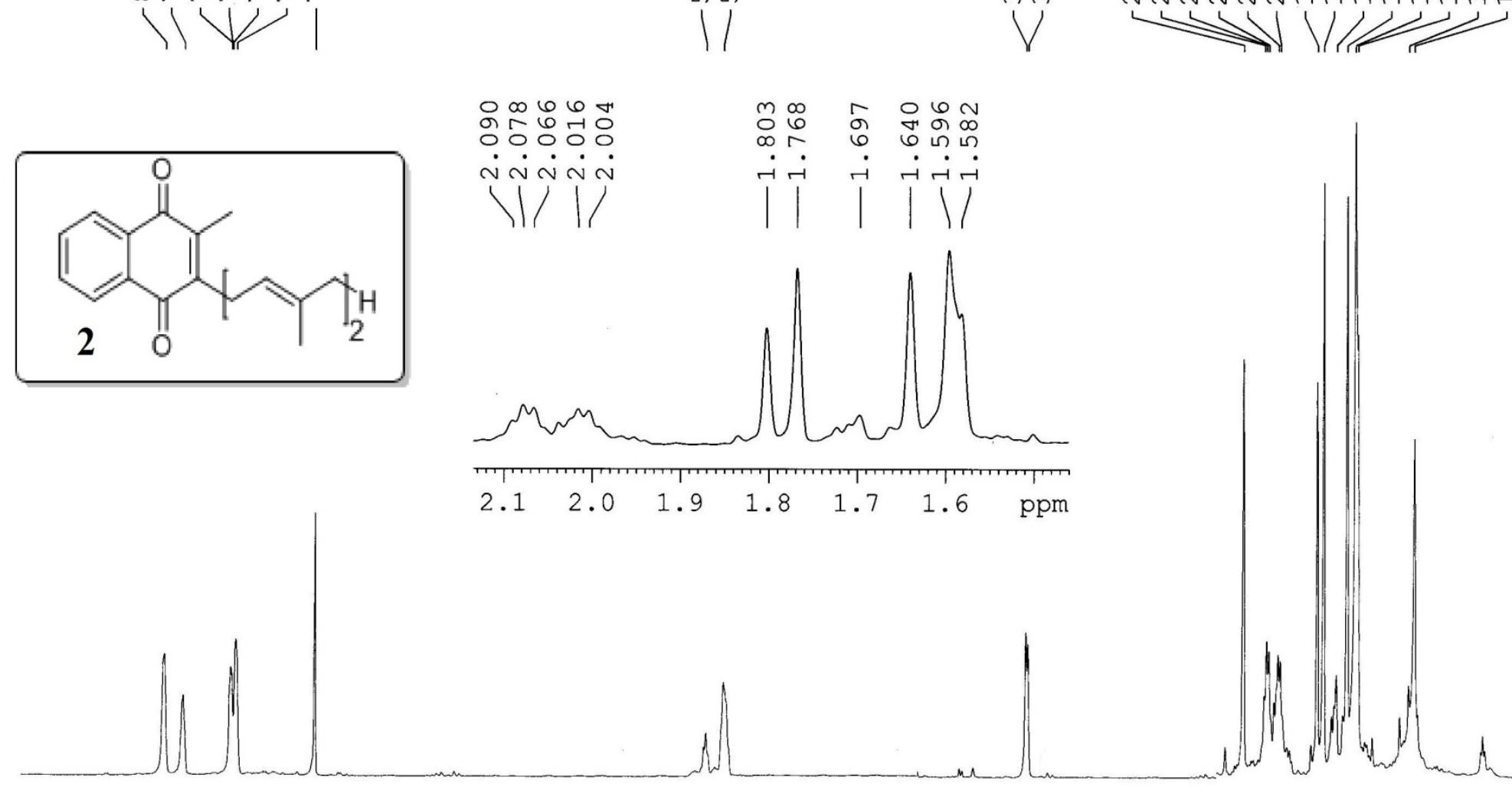

pm
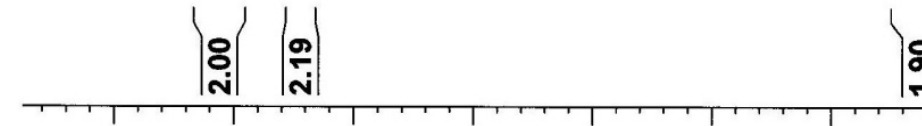

ฉి.

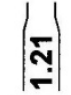

mall

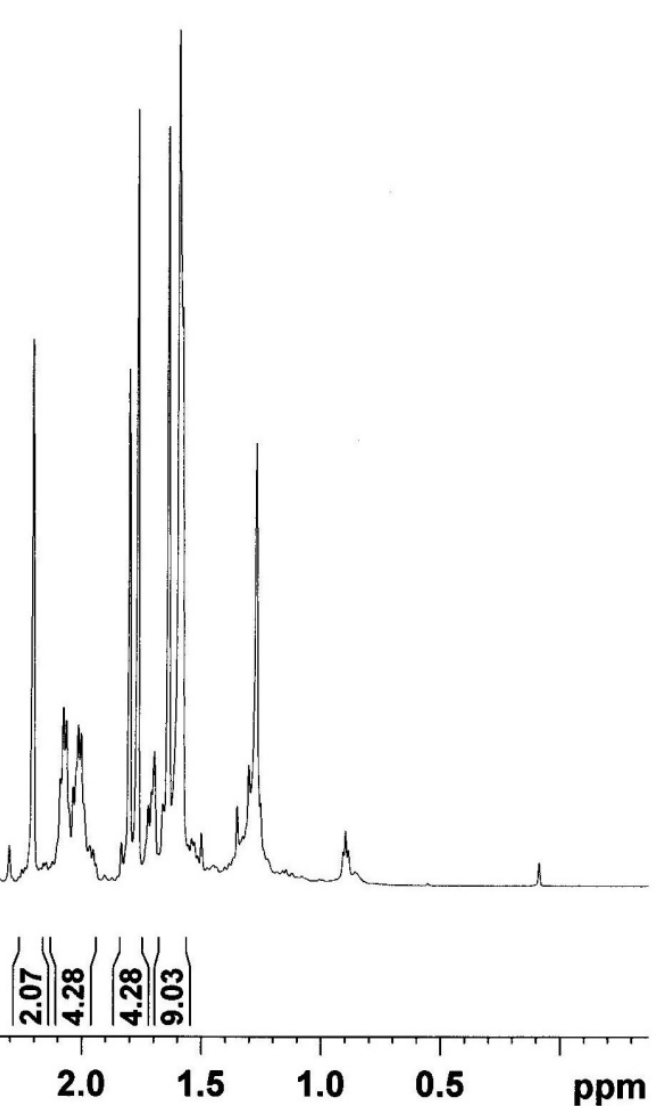




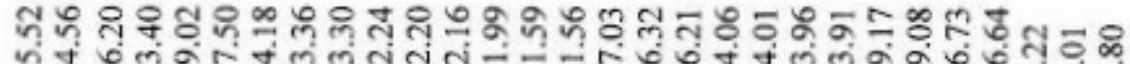

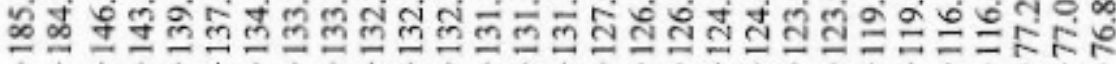
V

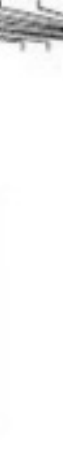

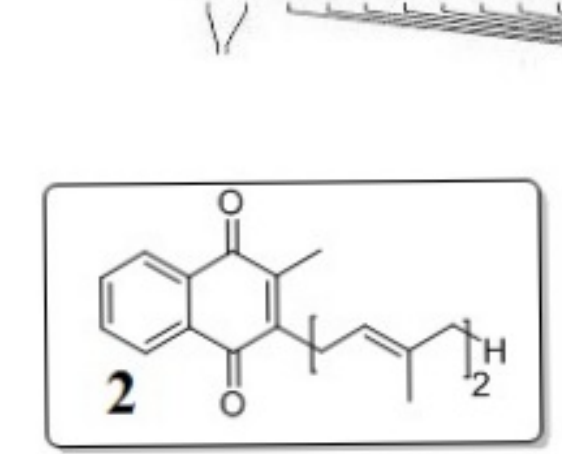

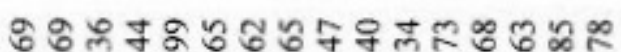

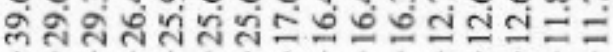

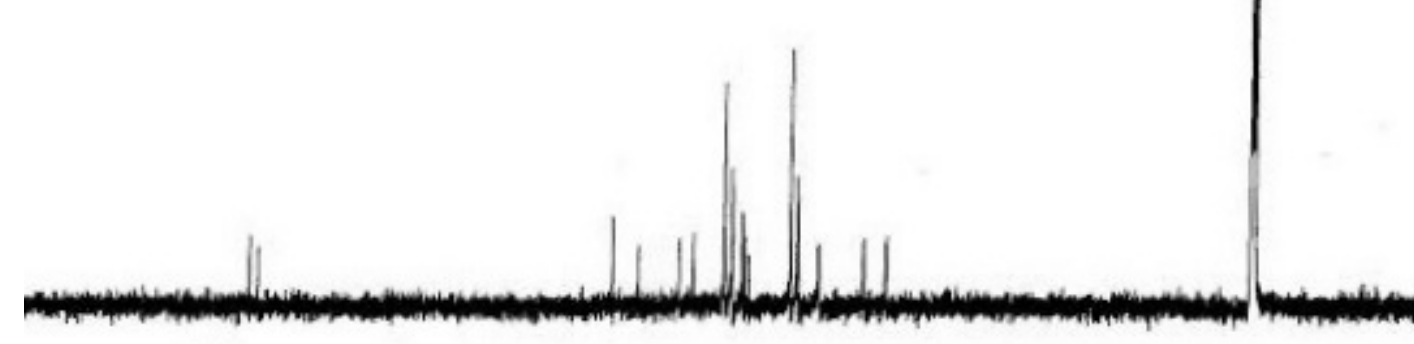

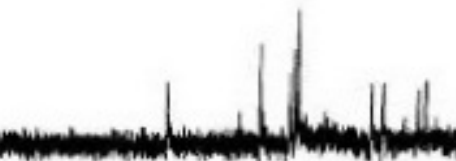
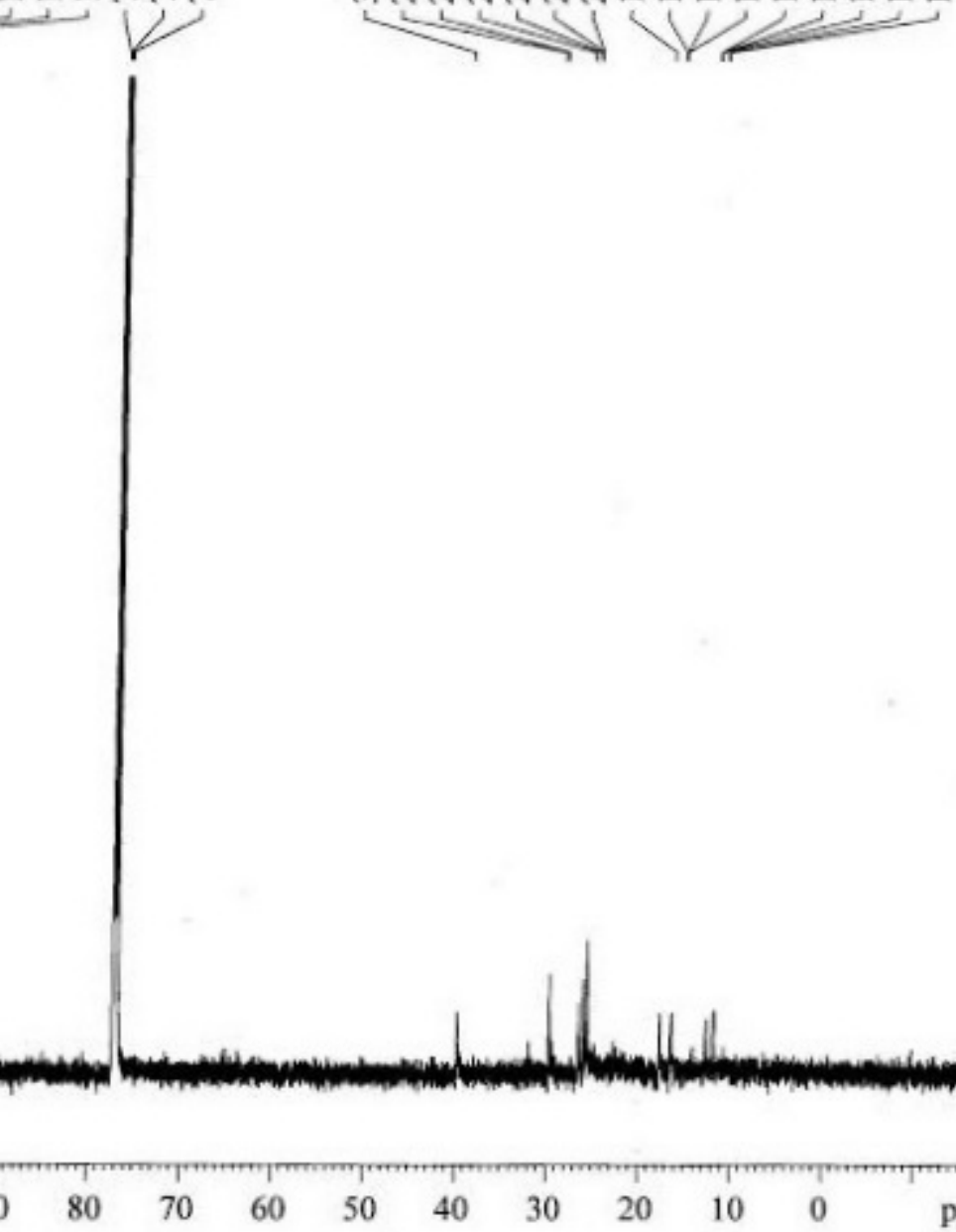

$70 \quad 60$

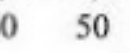

$\begin{array}{lll}40 & 30 & 20\end{array}$

100

ppm 
$\mathrm{dm} / \mathrm{kg} / 705-1 \mathrm{~h}$
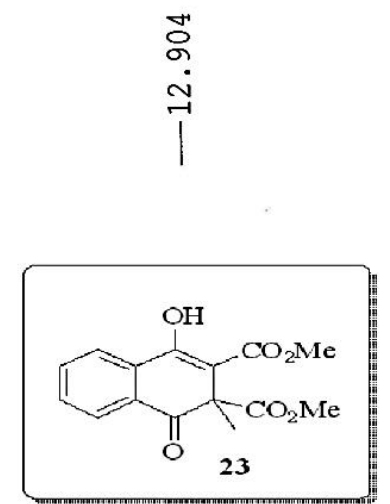

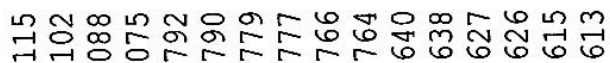

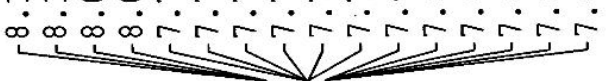

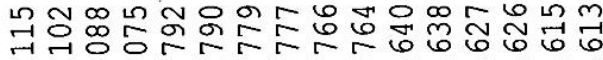

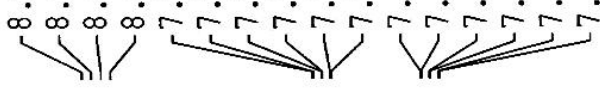
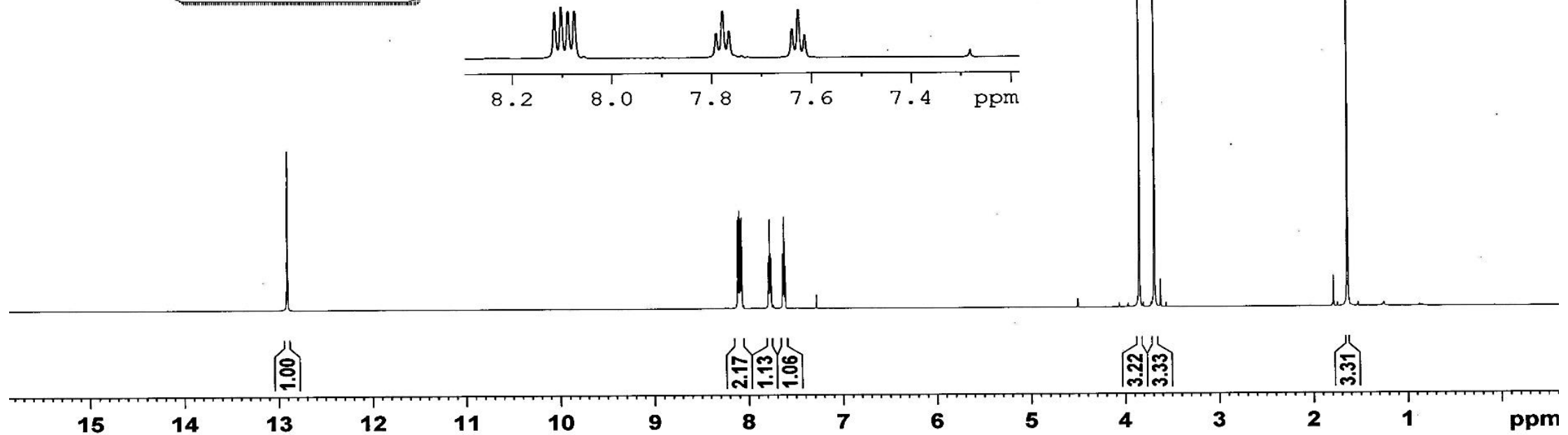
${ }^{13} \mathrm{C}$ NMR of compound 23 (CDCl3, $150 \mathrm{MHz}$ )

$\mathrm{dm} / \mathrm{kg} / 705-13 \mathrm{c}$
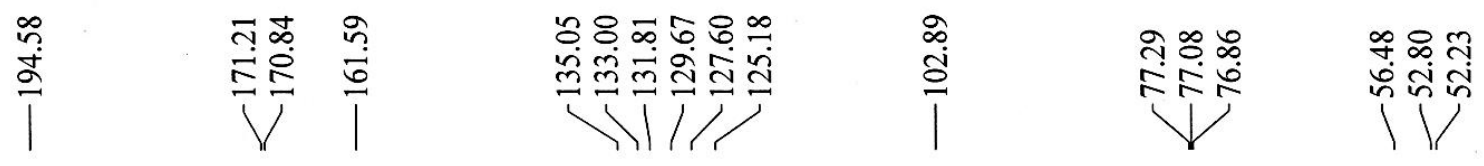

$\stackrel{2}{n}$
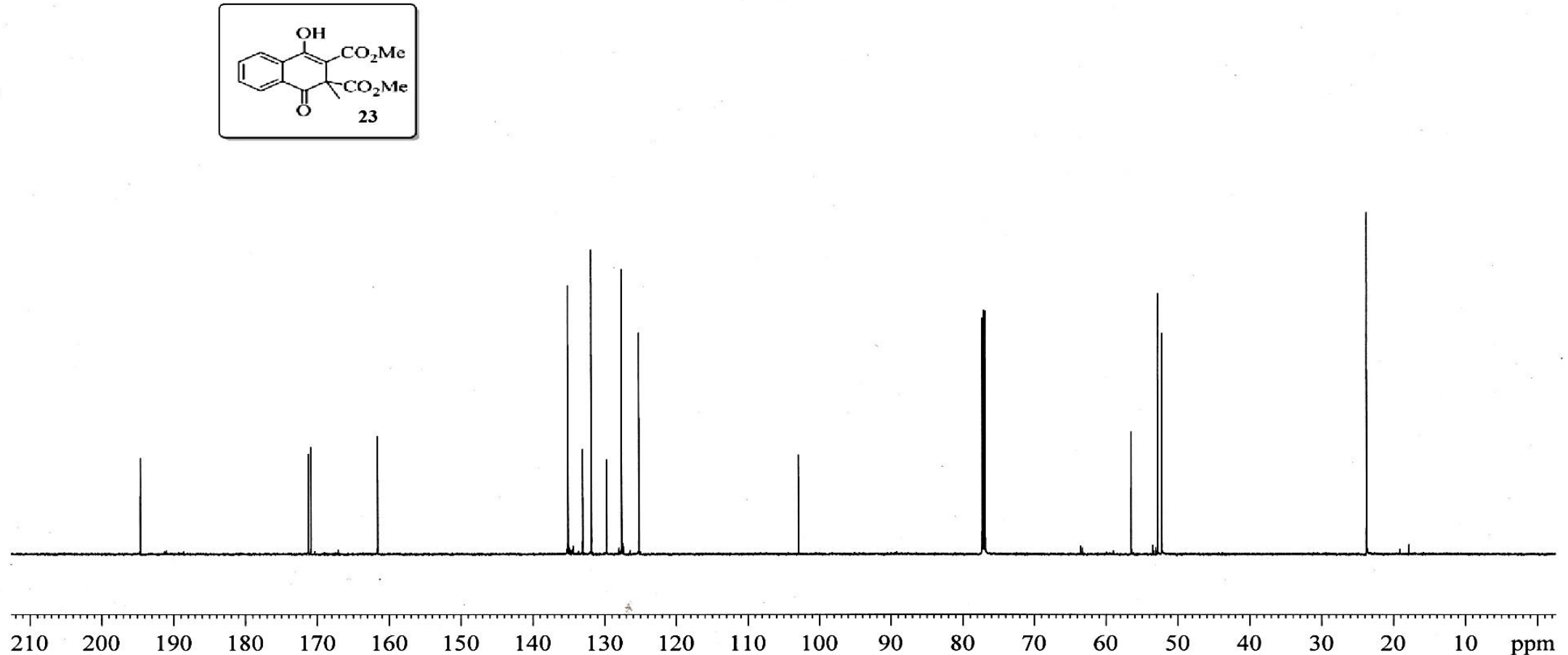
${ }^{1} \mathrm{H}$ NMR of compound $25\left(\mathrm{CDCl}_{3}, 600 \mathrm{MHz}\right)$

$\mathrm{dm} / \mathrm{kg} / 731-1 \mathrm{~h}$

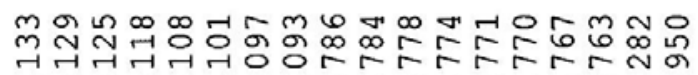

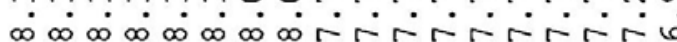

$\longrightarrow$

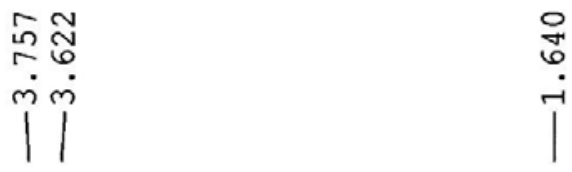

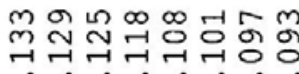

$\infty \dot{\infty} \infty \dot{\infty} \infty \dot{\infty} \infty \dot{\infty}$

$\rightarrow 1$

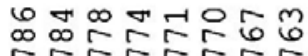

T.T. T. T.

3

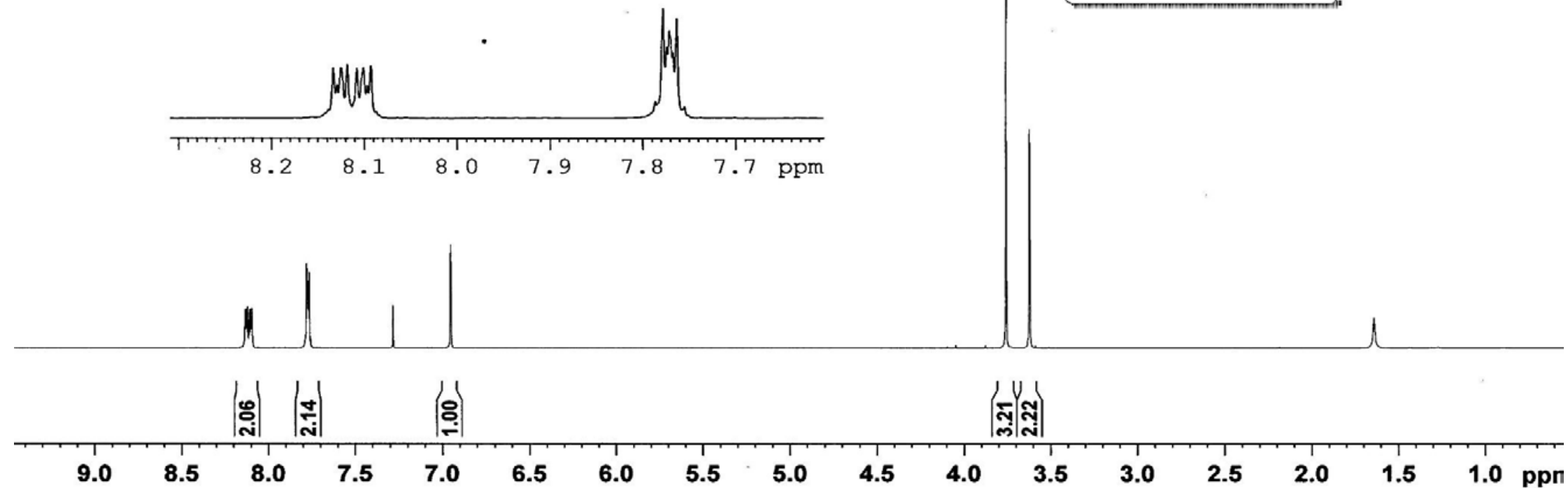


${ }^{13} \mathrm{C}$ NMR of compound $25\left(\mathrm{CDCl}_{3}, 150 \mathrm{MHz}\right)$

$\mathrm{dm} / \mathrm{kg} / 731-13 \mathrm{c}$

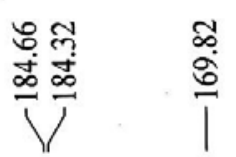

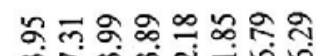

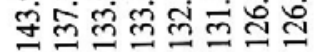

रा

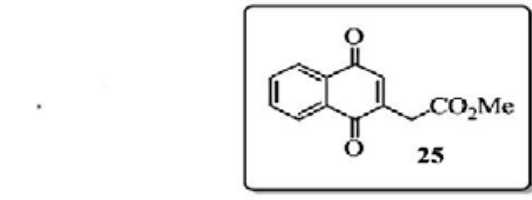

두유

।

i

.
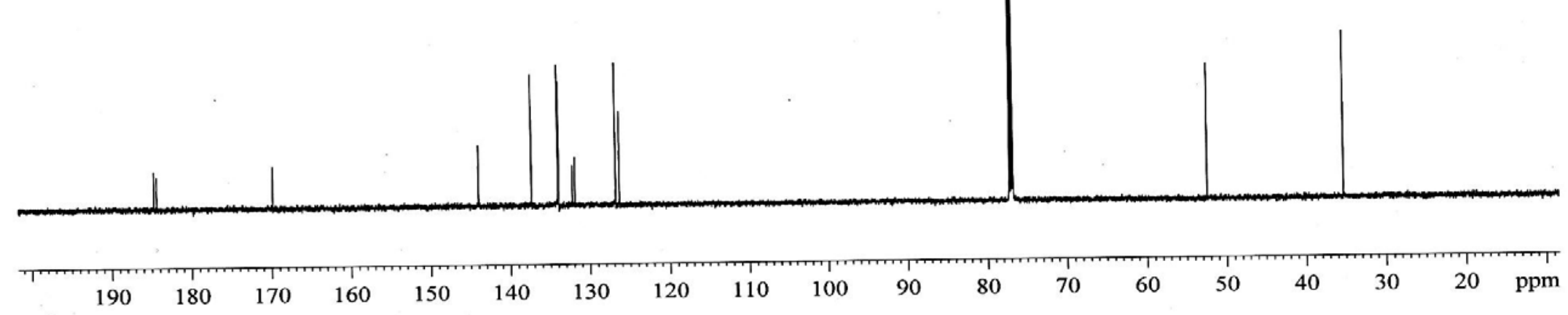
${ }^{1} \mathrm{H}$ NMR of compound 28c $\left(\mathrm{CDCl}_{3}, 600 \mathrm{MHz}\right)$

$\mathrm{dm} / \mathrm{sb} / 745-1 \mathrm{~h}$
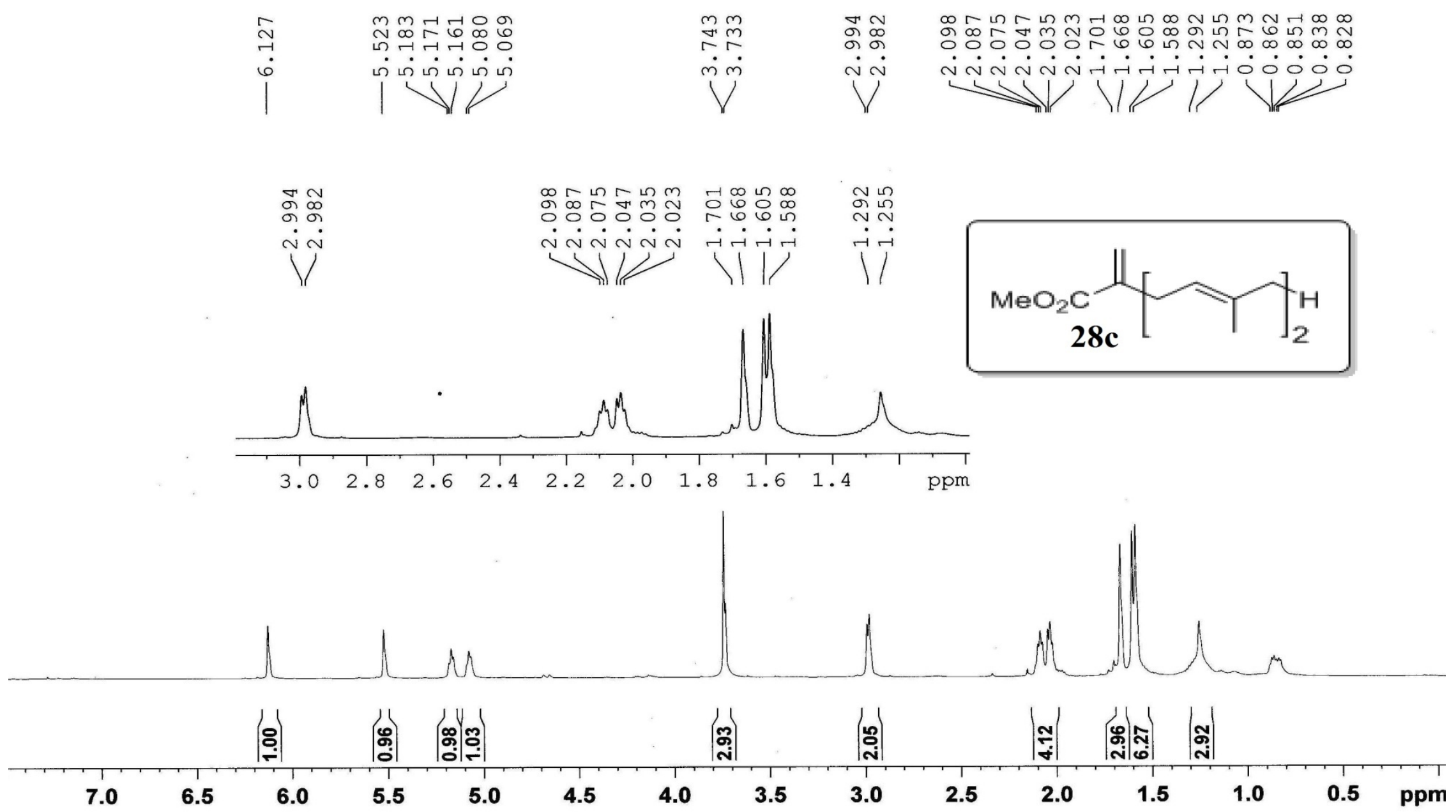
${ }^{13} \mathrm{C}$ NMR of compound 28c $\left(\mathrm{CDCl}_{3}, 150 \mathrm{MHz}\right)$

$\mathrm{dm} / \mathrm{kg} / 745-13 \mathrm{c}$

\begin{tabular}{|c|c|c|c|c|c|}
\hline$\underset{\tilde{i}}{\stackrel{2}{6}}$ & 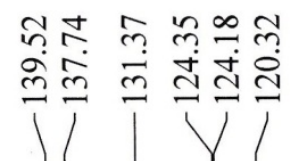 & 㛋 & $\begin{array}{l}\infty \\
\dot{n}\end{array}$ & $\begin{array}{l}\hat{\sigma} \\
\text { ⿵ें }\end{array}$ & 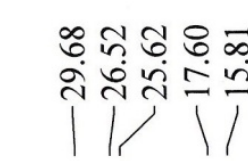 \\
\hline
\end{tabular}
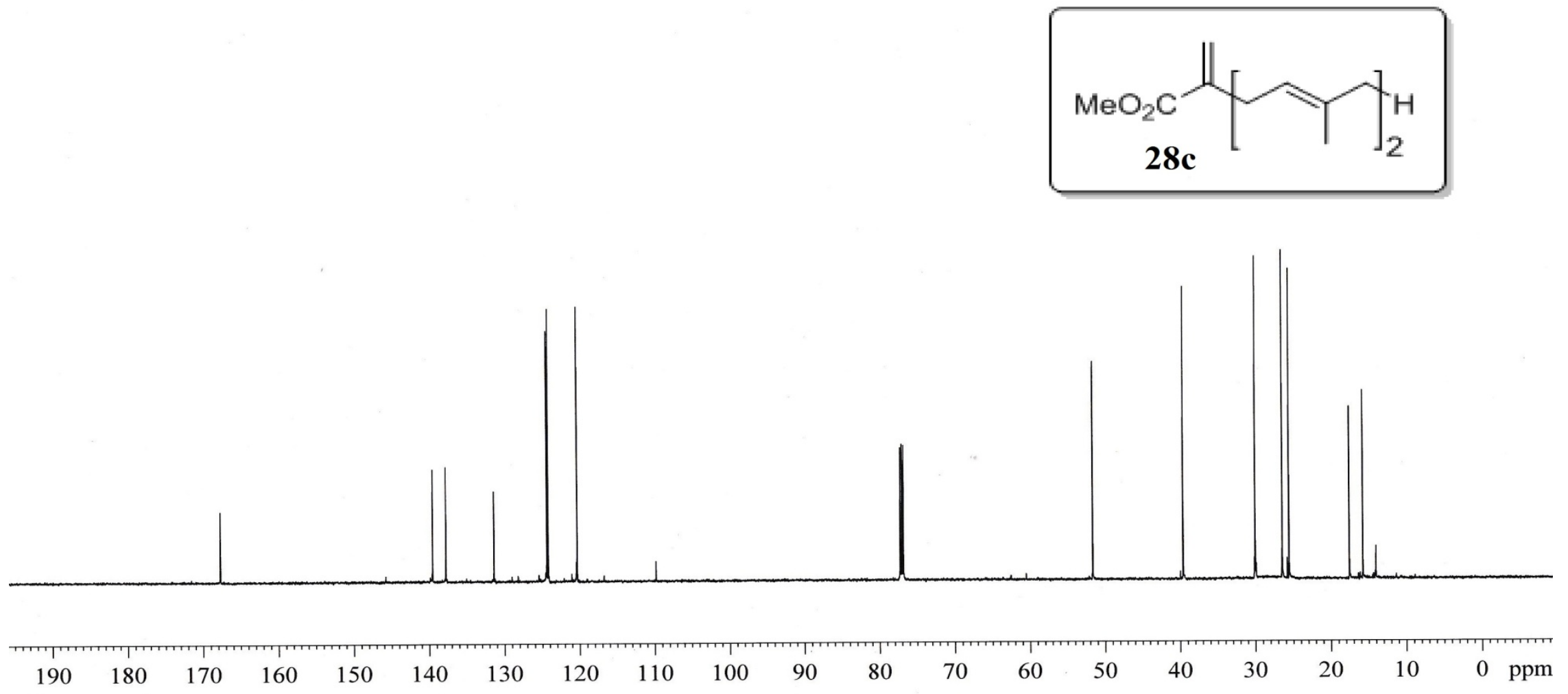
${ }^{1} \mathrm{H}$ NMR of compound 28d $\left(\mathrm{CDCl}_{3}, 600 \mathrm{MHz}\right)$

/sj / $421-1 \mathrm{~h}-\mathrm{f} 581$

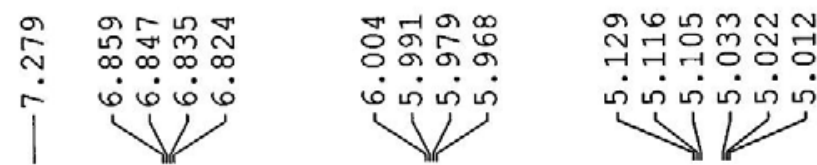

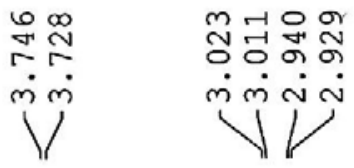

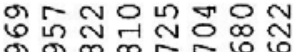

نं

iंi

vili
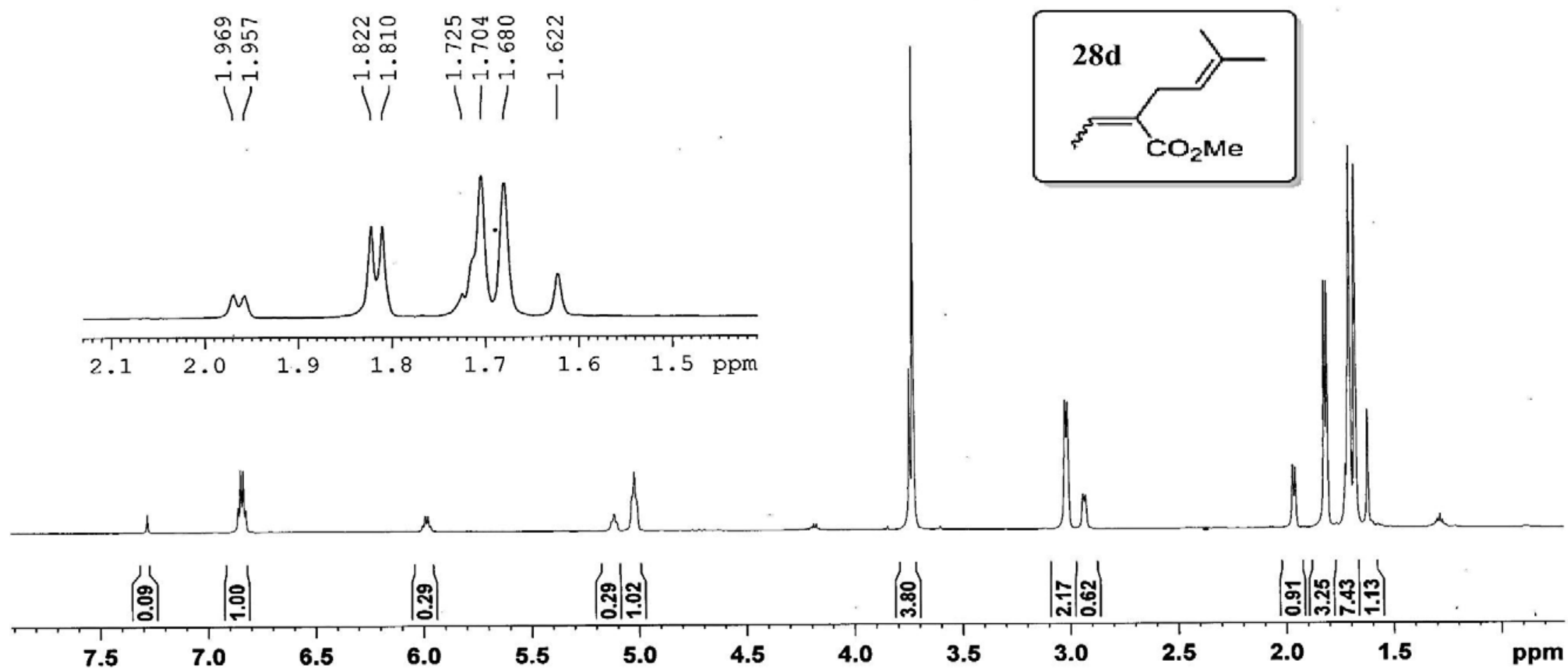
${ }^{13} \mathrm{C}$ NMR of compound 28d ( $\left.\mathrm{CDCl}_{3}, 150 \mathrm{MHz}\right)$
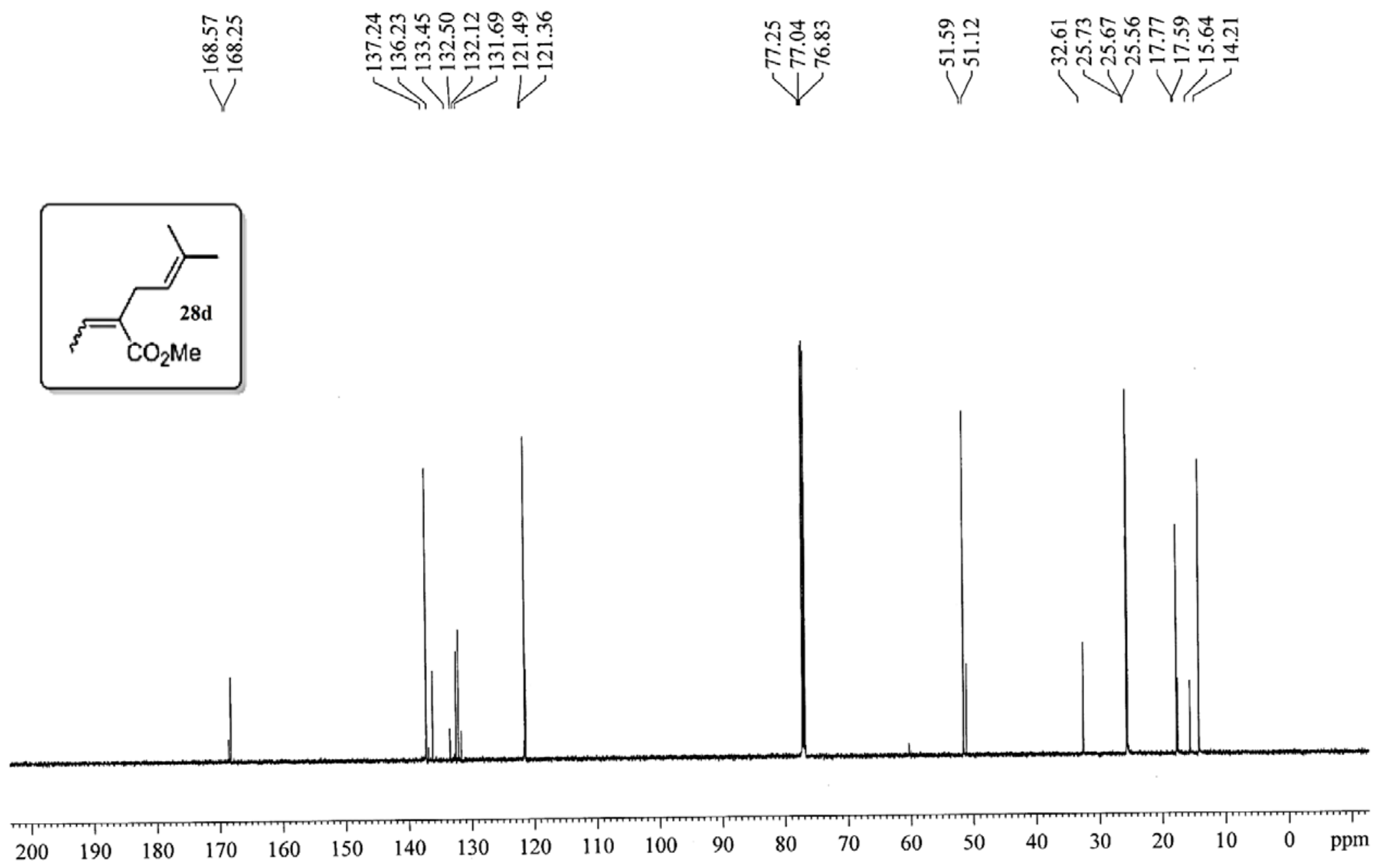
${ }^{1} \mathrm{H}$ NMR of compound $28 \mathrm{e}\left(\mathrm{CDCl}_{3}, 600 \mathrm{MHz}\right)$

$\mathrm{dm} / \mathrm{sj} / 423 \mathrm{col}-1 \mathrm{~h}$

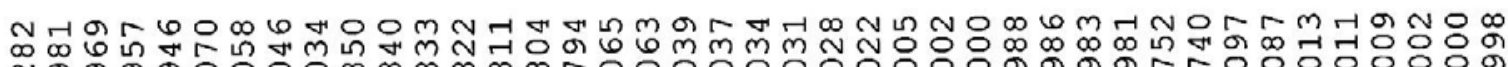
N \%̆ ن

눙용 $\circ$ o $\infty \infty$

N $-1-1-1$

Vi

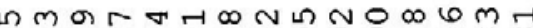

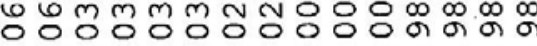
10 $\rightarrow 1 / 1 / 1 / 1$
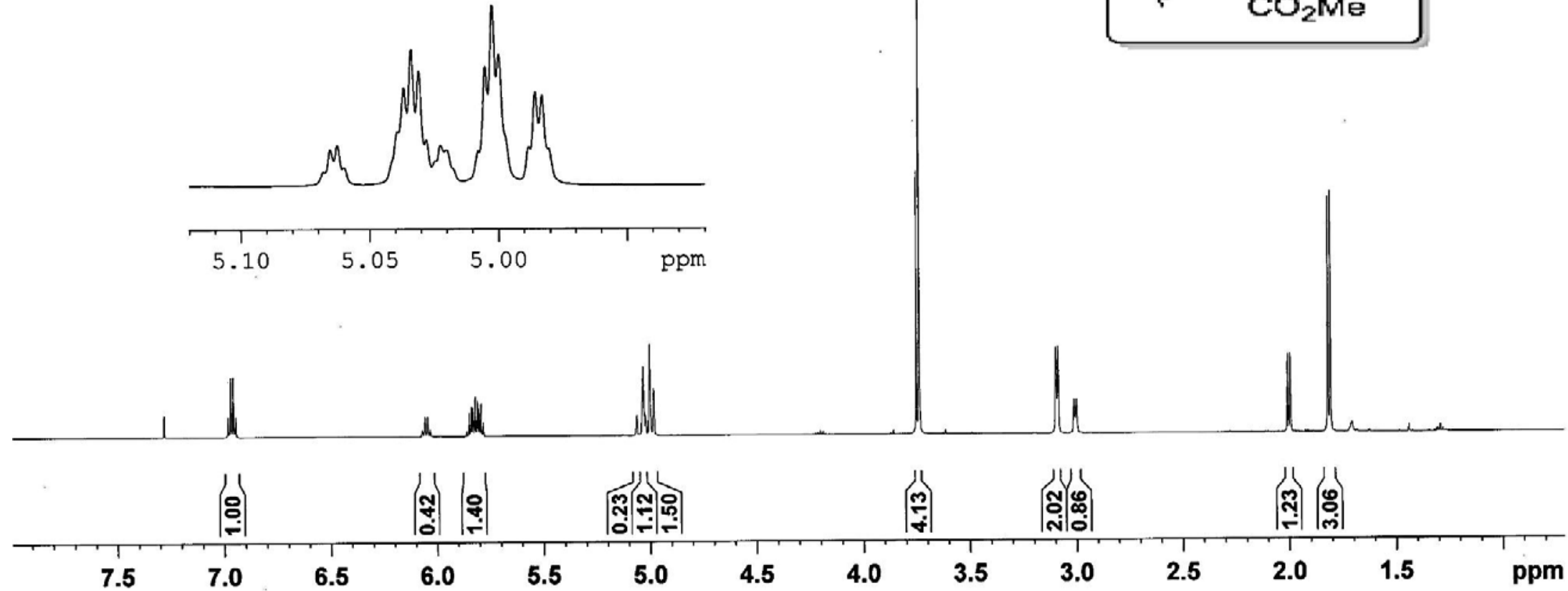
${ }^{13} \mathrm{C}$ NMR of compound 28e $\left(\mathrm{CDCl}_{3}, 150 \mathrm{MHz}\right)$

\begin{tabular}{|c|c|c|c|c|c|c|}
\hline 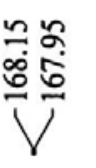 & 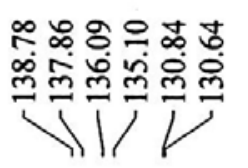 & $\frac{8}{1 /}$ & ฟ & 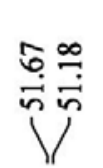 & ্ָণি & 管 \\
\hline
\end{tabular}
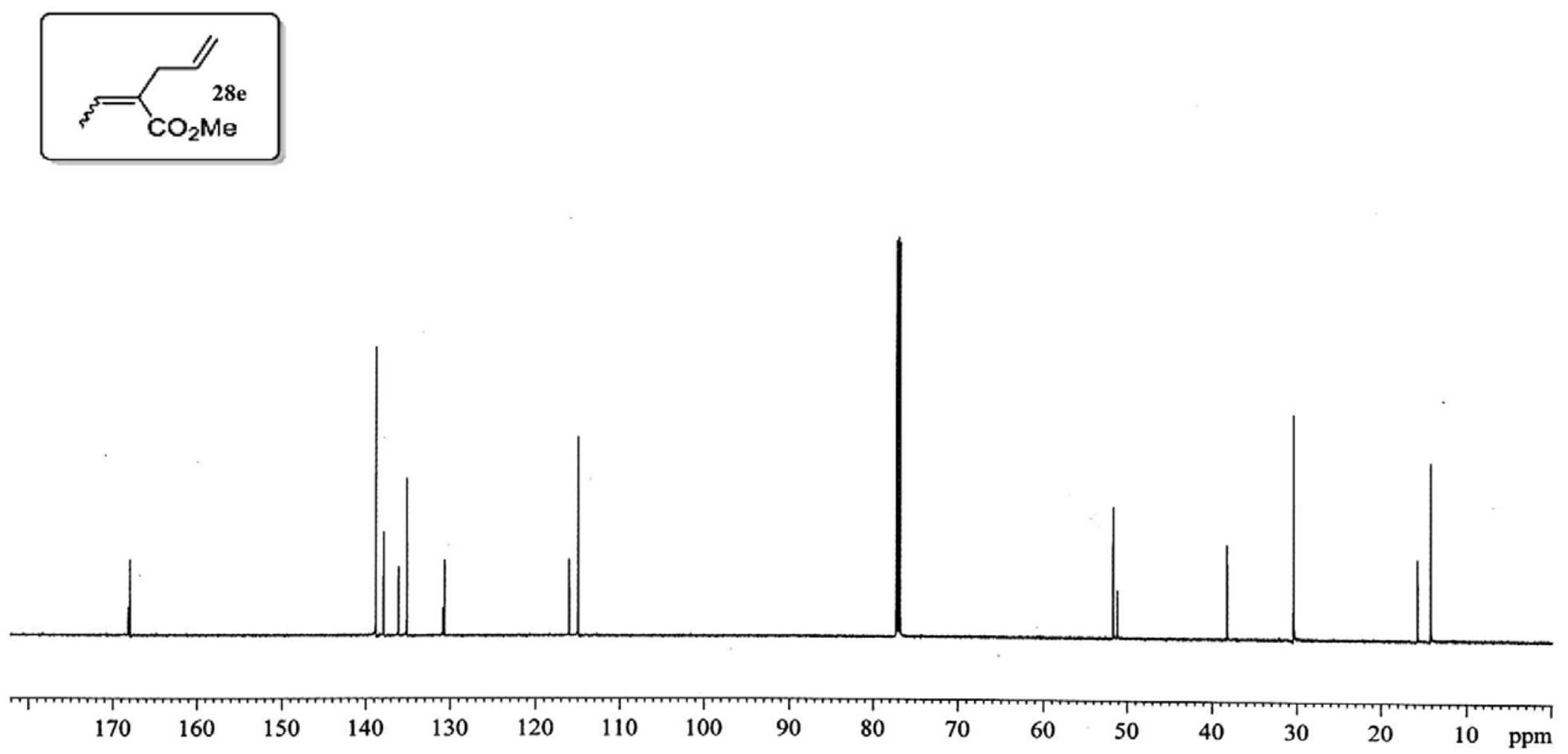
${ }^{1} \mathrm{H}$ NMR of compound $28 f\left(\mathrm{CDCl}_{3}, 600 \mathrm{MHz}\right)$

$\mathrm{dm} / \mathrm{sj} / 432 \mathrm{c}-1 \mathrm{~h}$

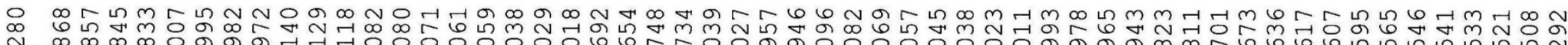

0 60 in

1

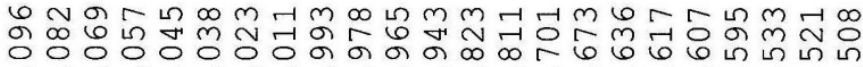

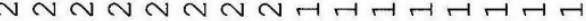

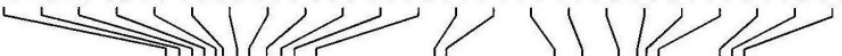
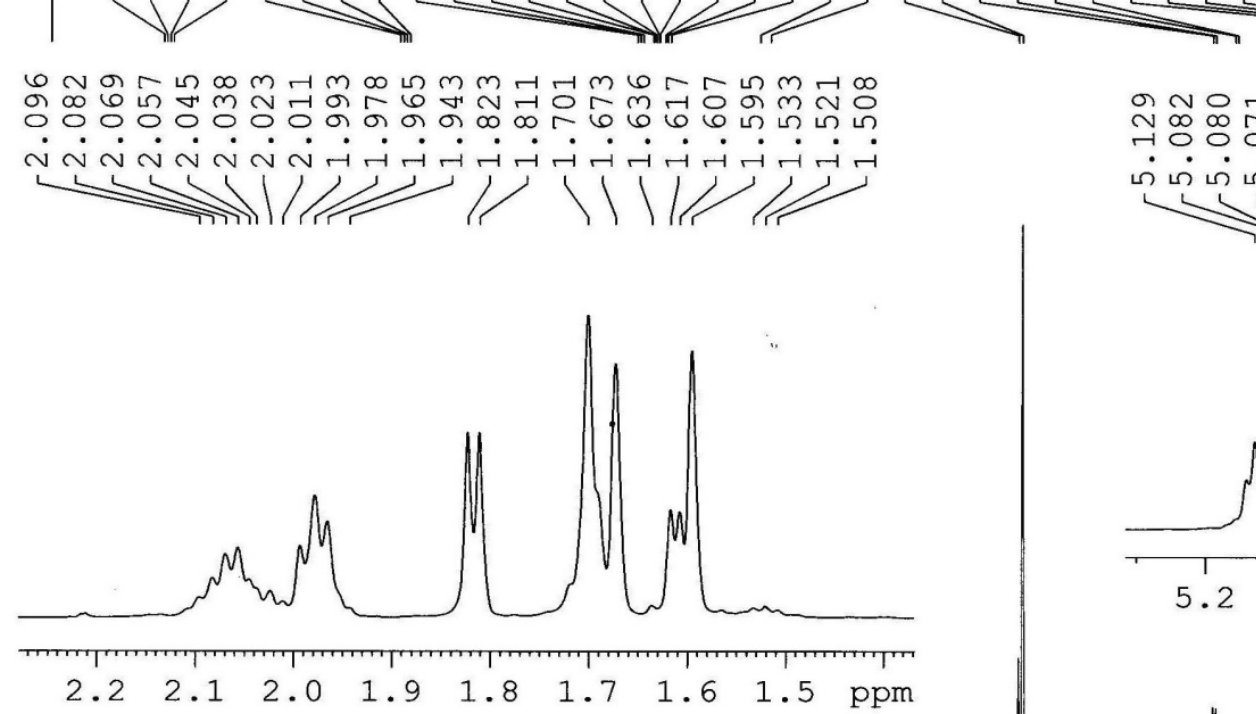

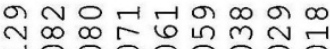

ن்
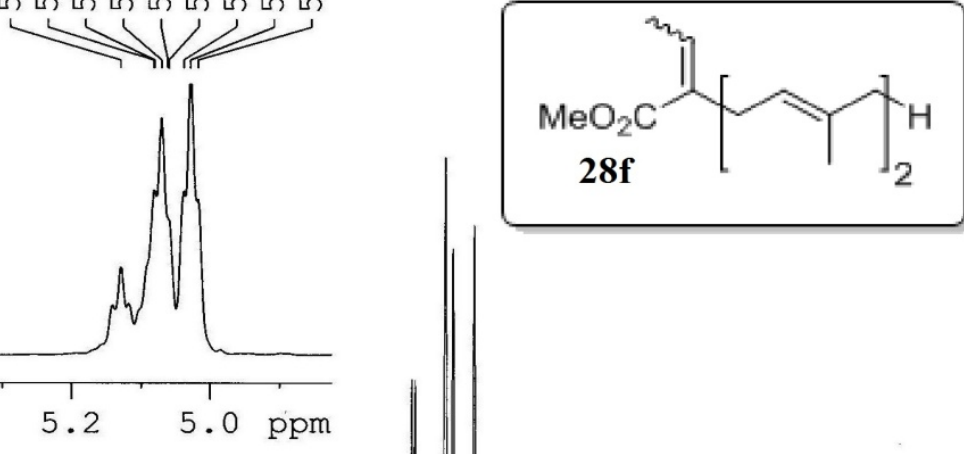

$\begin{array}{llllllllll}2.2 & 2.1 & 2.0 & 1.9 & 1.8 & 1.7 & 1.6 & 1.5 & \mathrm{ppm}\end{array}$

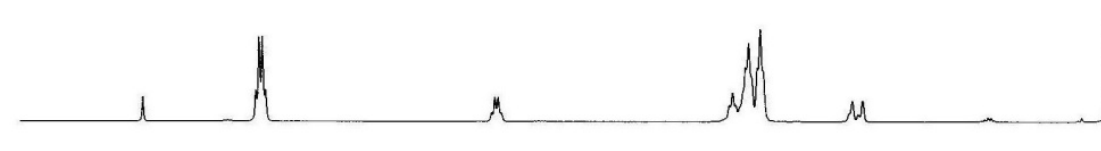

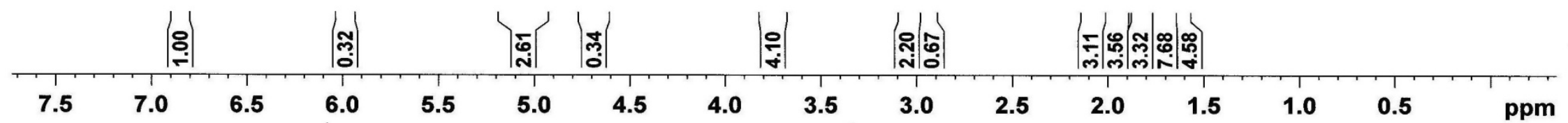




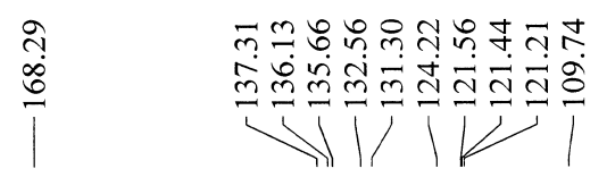

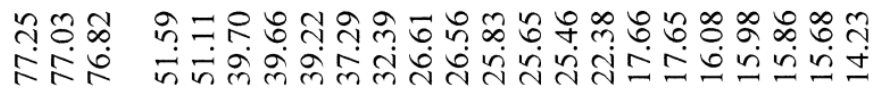
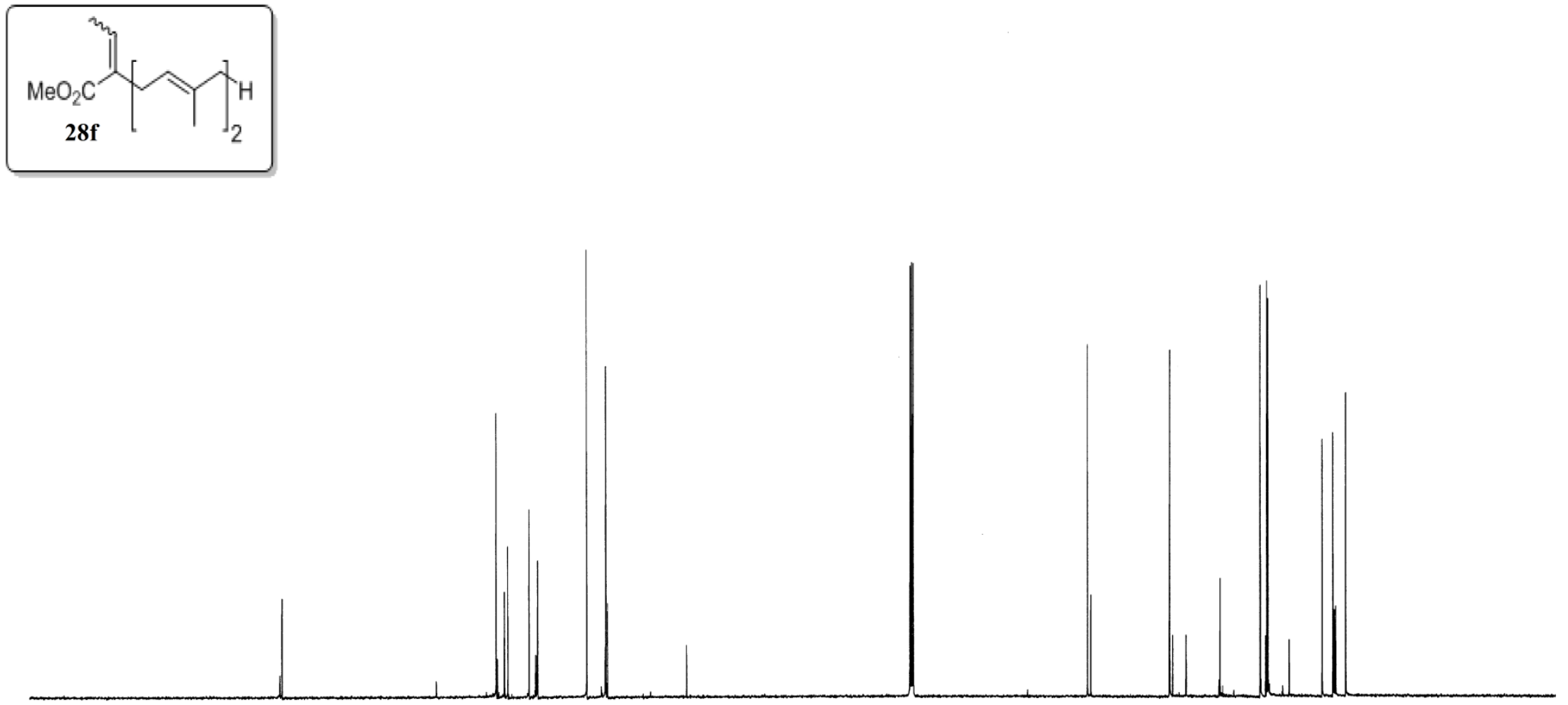$$
\text { गाम }
$$

$0 \quad 160$

$\begin{array}{lll}150 & 140 \quad 130\end{array}$

120

10100

90

$80 \quad 70$

60

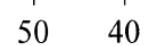

$40 \quad 30$

20

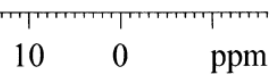


${ }^{1} \mathrm{H}$ NMR of compound 31a $\left(\mathrm{CDCl}_{3}, 600 \mathrm{MHz}\right)$

$/ \mathrm{kg} / 732-1 \mathrm{~h}$

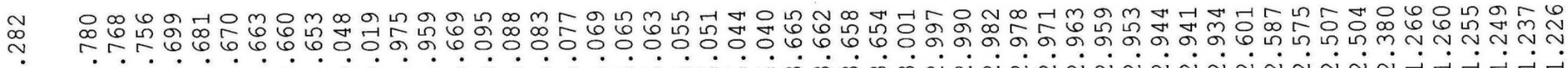
r

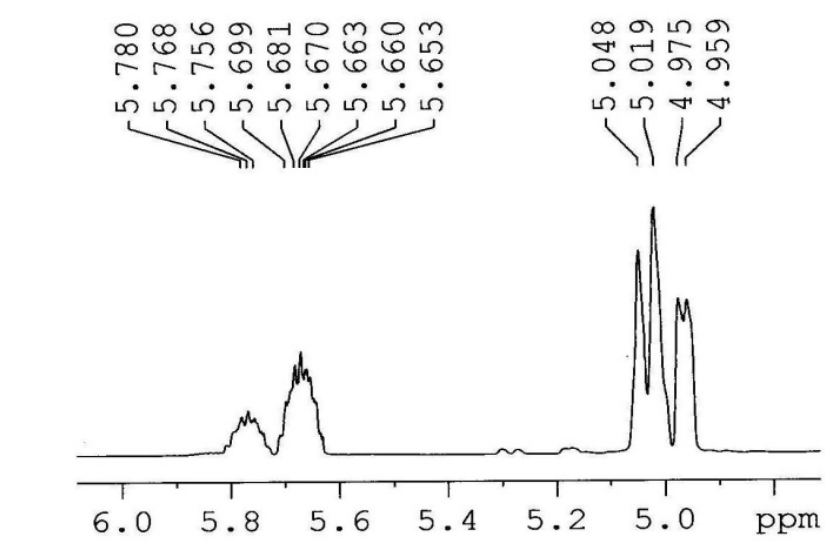

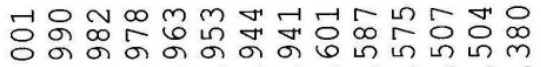
$\dot{\sim} \dot{\sim} \dot{\sim} \dot{\sim} \dot{\sim} \dot{\sim} \dot{\sim} \dot{\sim}$

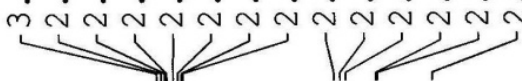
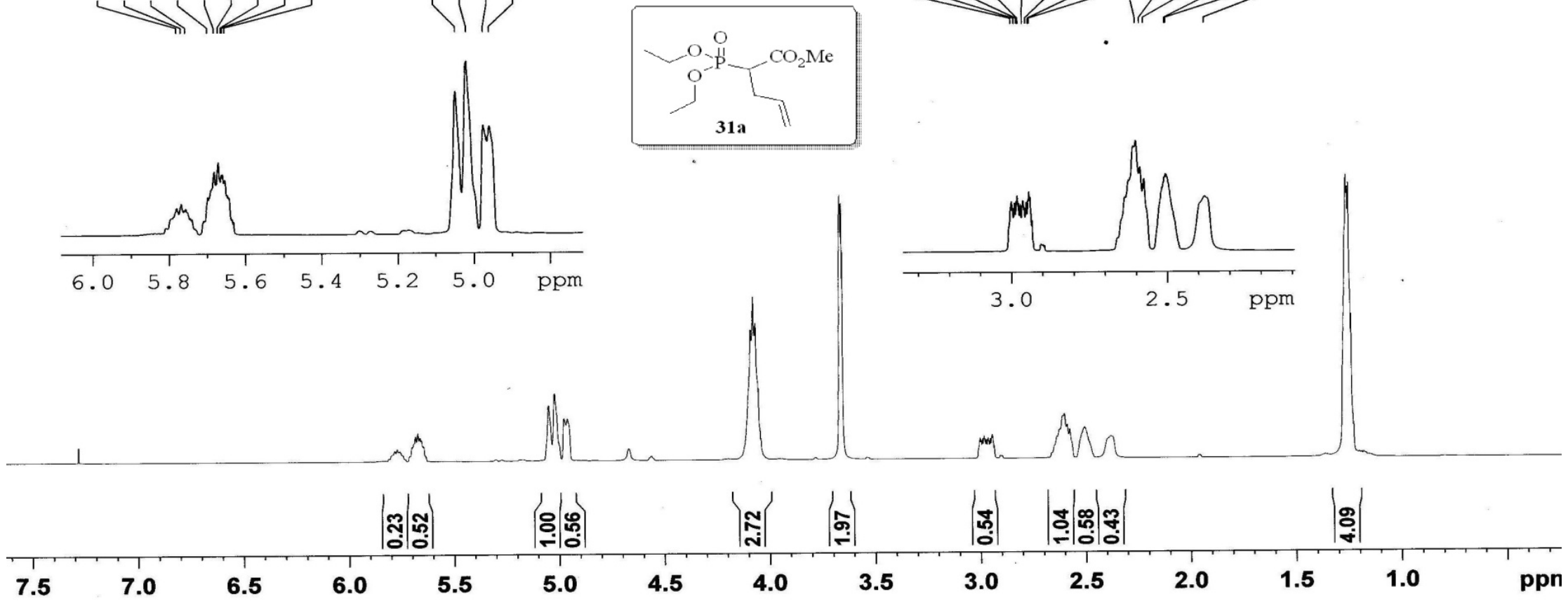
${ }^{13} \mathrm{C}$ NMR of compound 31a $\left(\mathrm{CDCl}_{3}, 150 \mathrm{MHz}\right)$

$\mathrm{dm} / \mathrm{kg} / 732-13 \mathrm{c}$

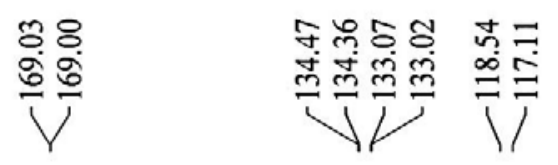

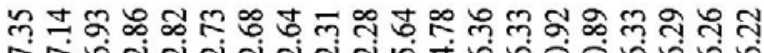

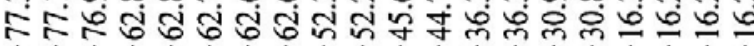

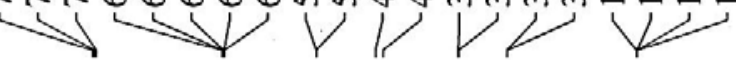
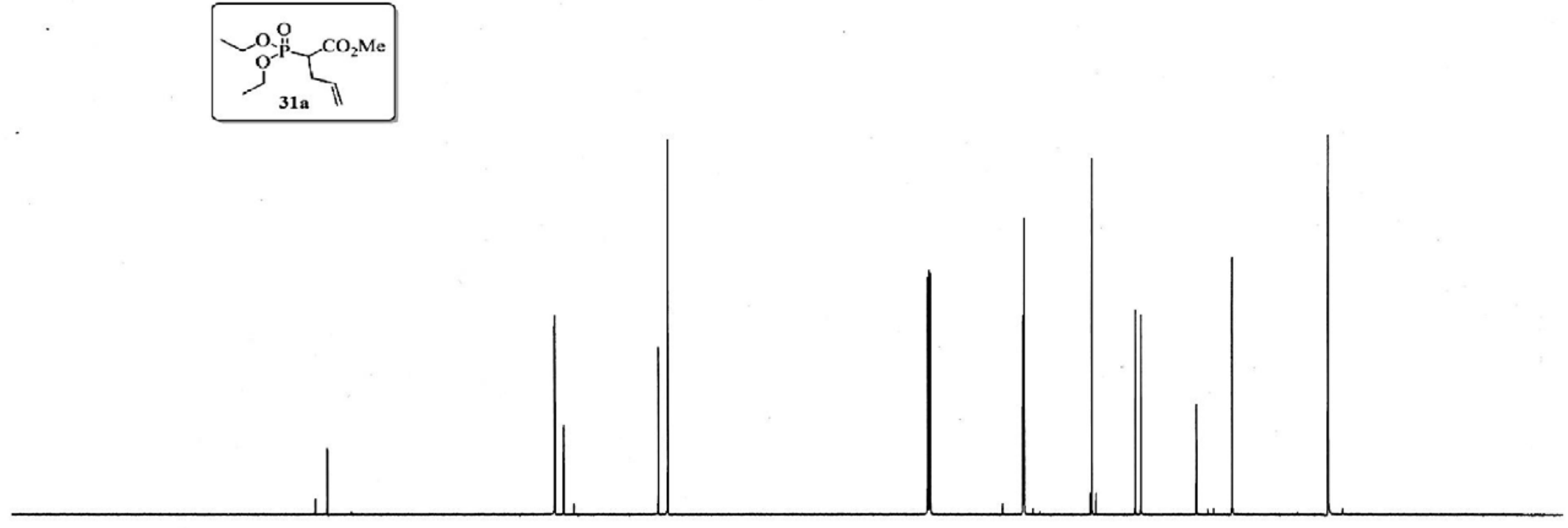

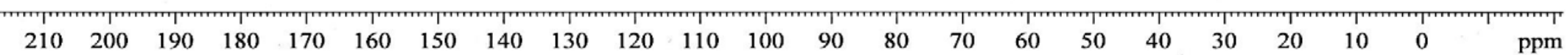

210200
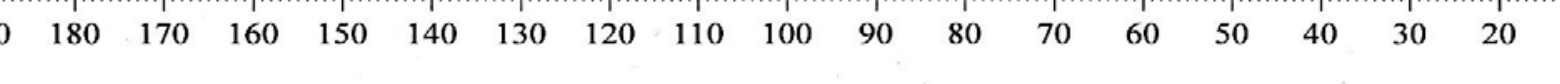

ppm 


\section{${ }^{1} \mathrm{H}$ NMR of compound $43\left(\mathrm{CDCl}_{3}, 600 \mathrm{MHz}\right)$}

-......, ........
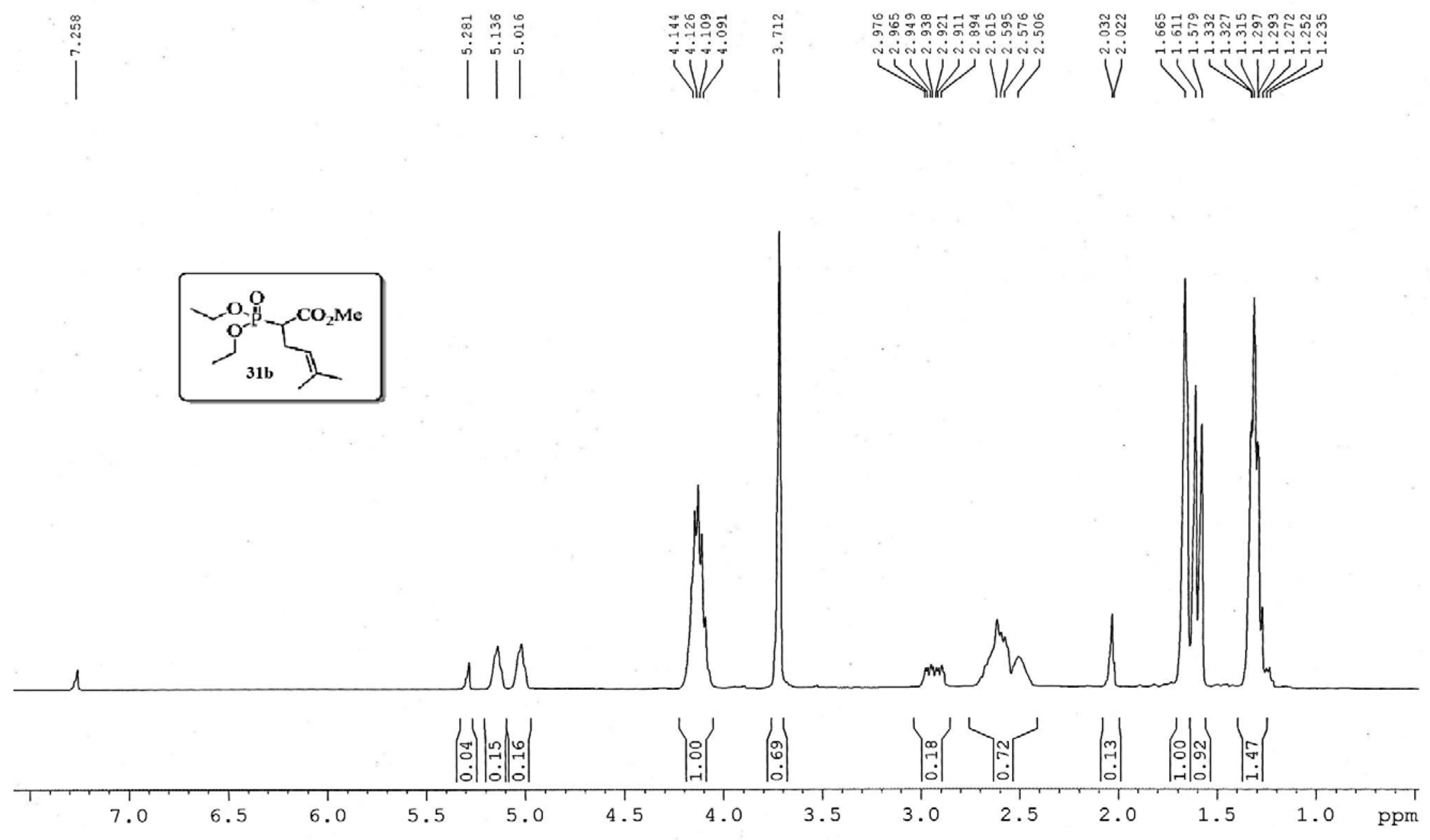
${ }^{13} \mathrm{C}$ NMR of compound 31b $\left(\mathrm{CDCl}_{3}, 150 \mathrm{MHz}\right)$

/ kg / 686pure - 13c
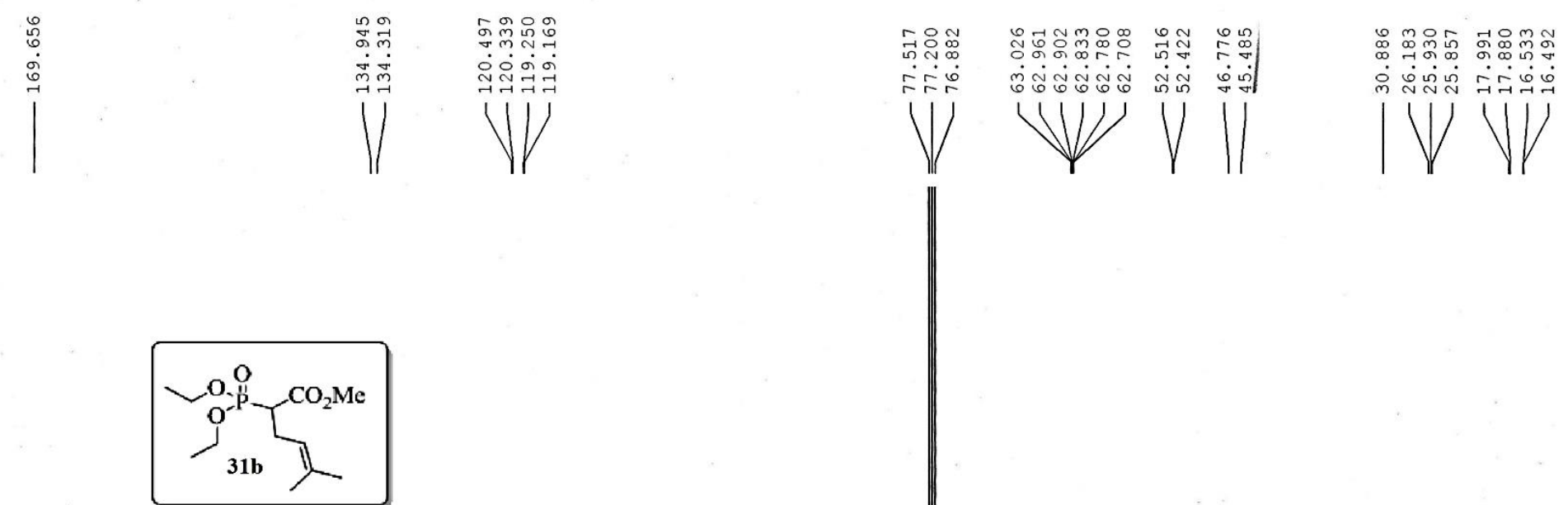

(N)

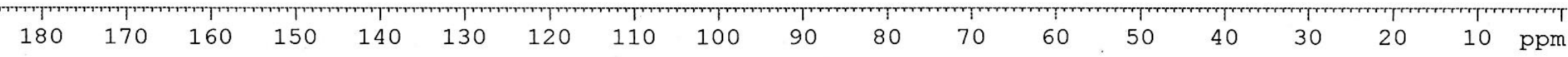


${ }^{1} \mathrm{H}$ NMR of compound 31c $\left(\mathrm{CDCl}_{3}, 600 \mathrm{MHz}\right)$

$\mathrm{dm} / \mathrm{kg} / 744-1 \mathrm{~h}$

-

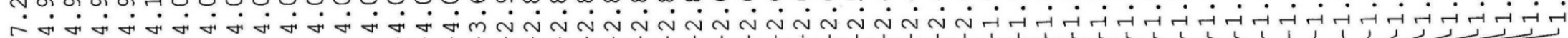

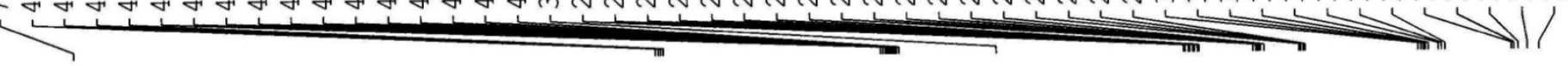

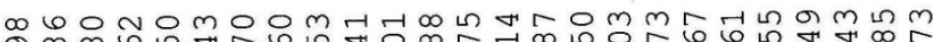

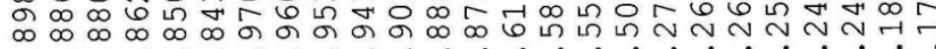
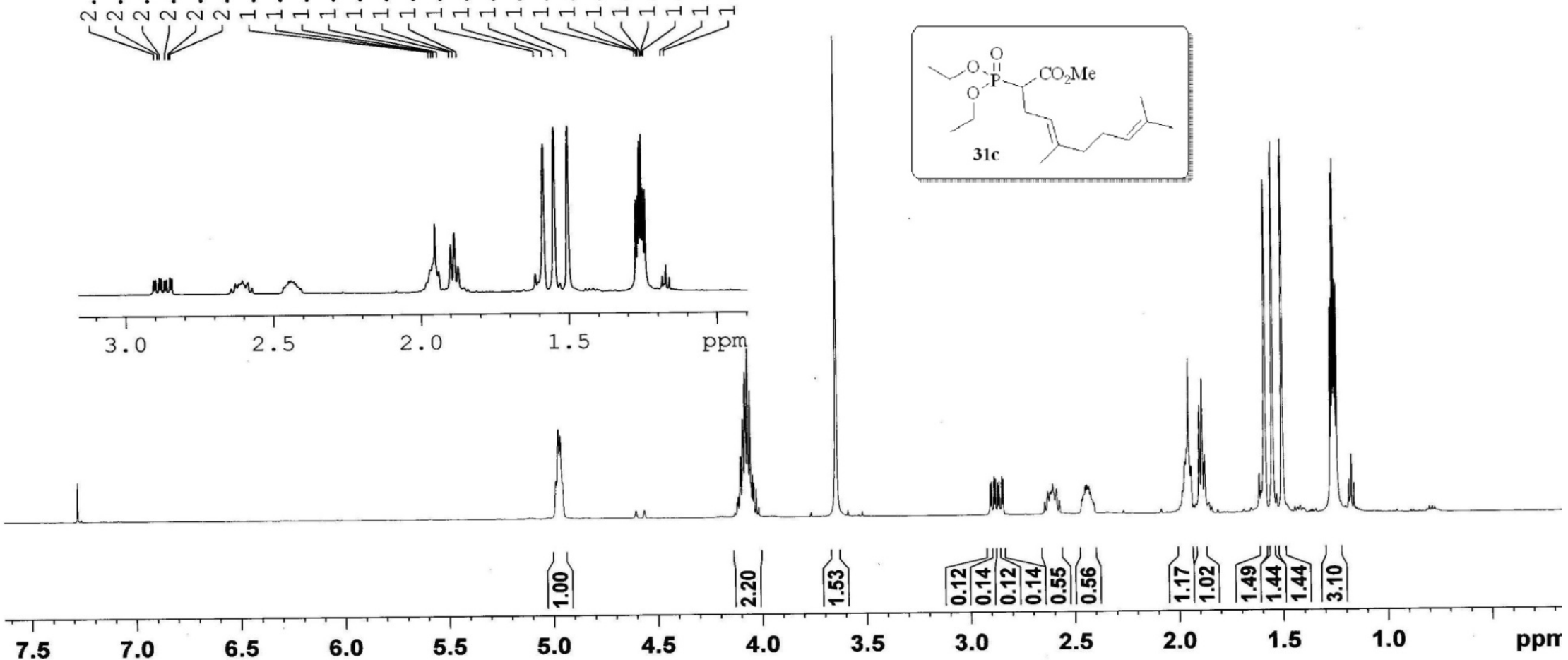
${ }^{13} \mathrm{C}$ NMR of compound 31c $\left(\mathrm{CDCl}_{3}, 150 \mathrm{MHz}\right)$

$\mathrm{dm} / \mathrm{kg} / 744-13 \mathrm{c}$

$$
\text { กิ }
$$

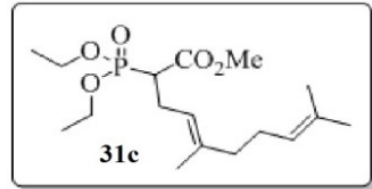

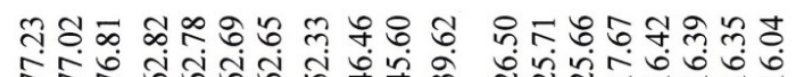

i)
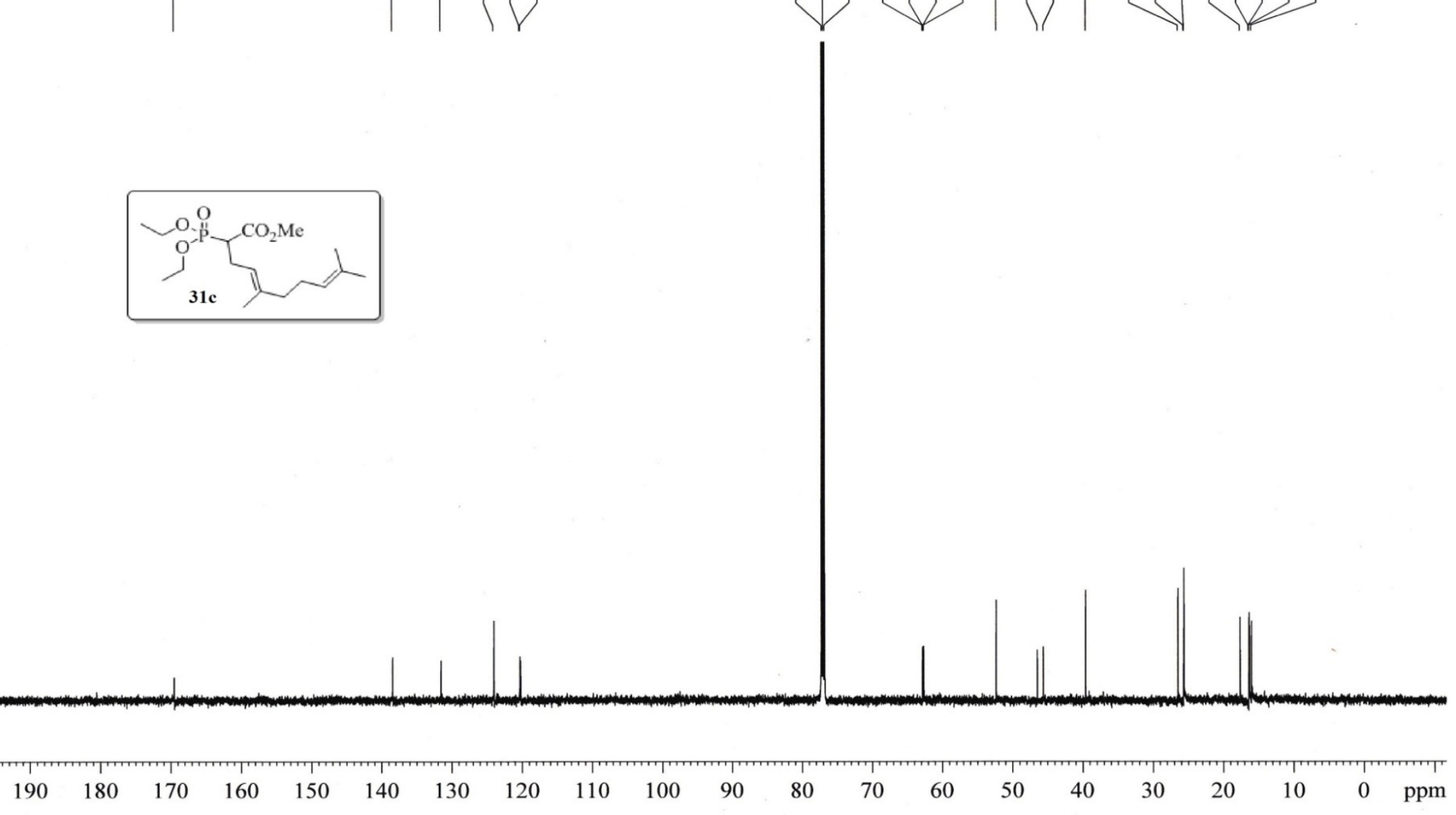
${ }^{1} \mathrm{H}$ NMR of compound $32\left(\mathrm{CDCl}_{3}, 600 \mathrm{MHz}\right)$

$\mathrm{dm} / \mathrm{kg} / 693-1 \mathrm{~h}$
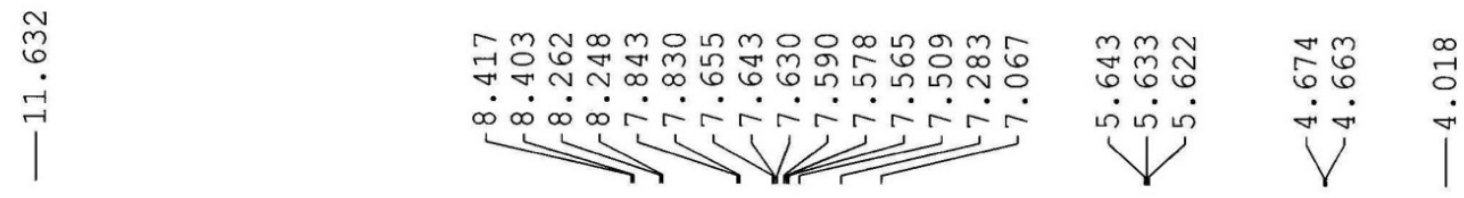

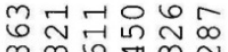
ર્ના

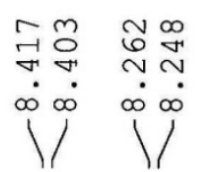

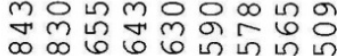

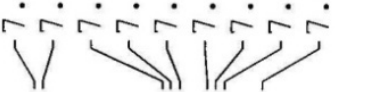
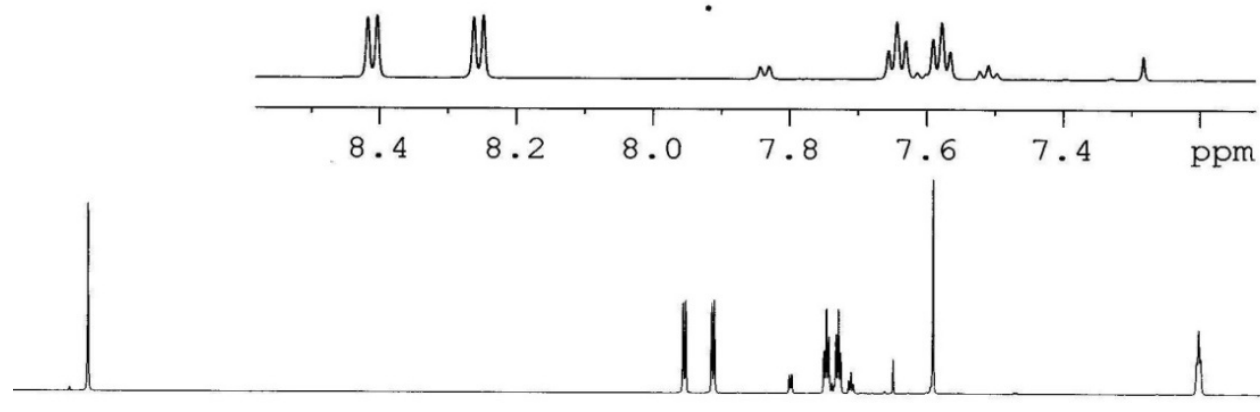

余

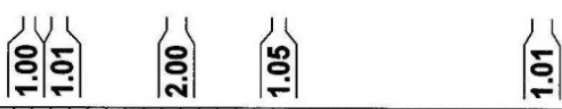

6

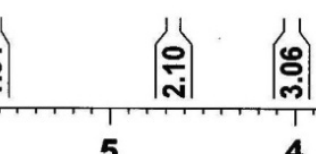

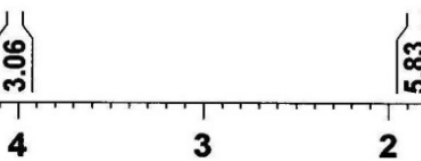

ח

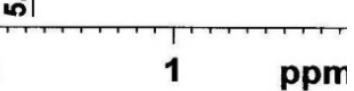


${ }^{13} \mathrm{C}$ NMR of compound $32\left(\mathrm{CDCl}_{3}, 150 \mathrm{MHz}\right)$

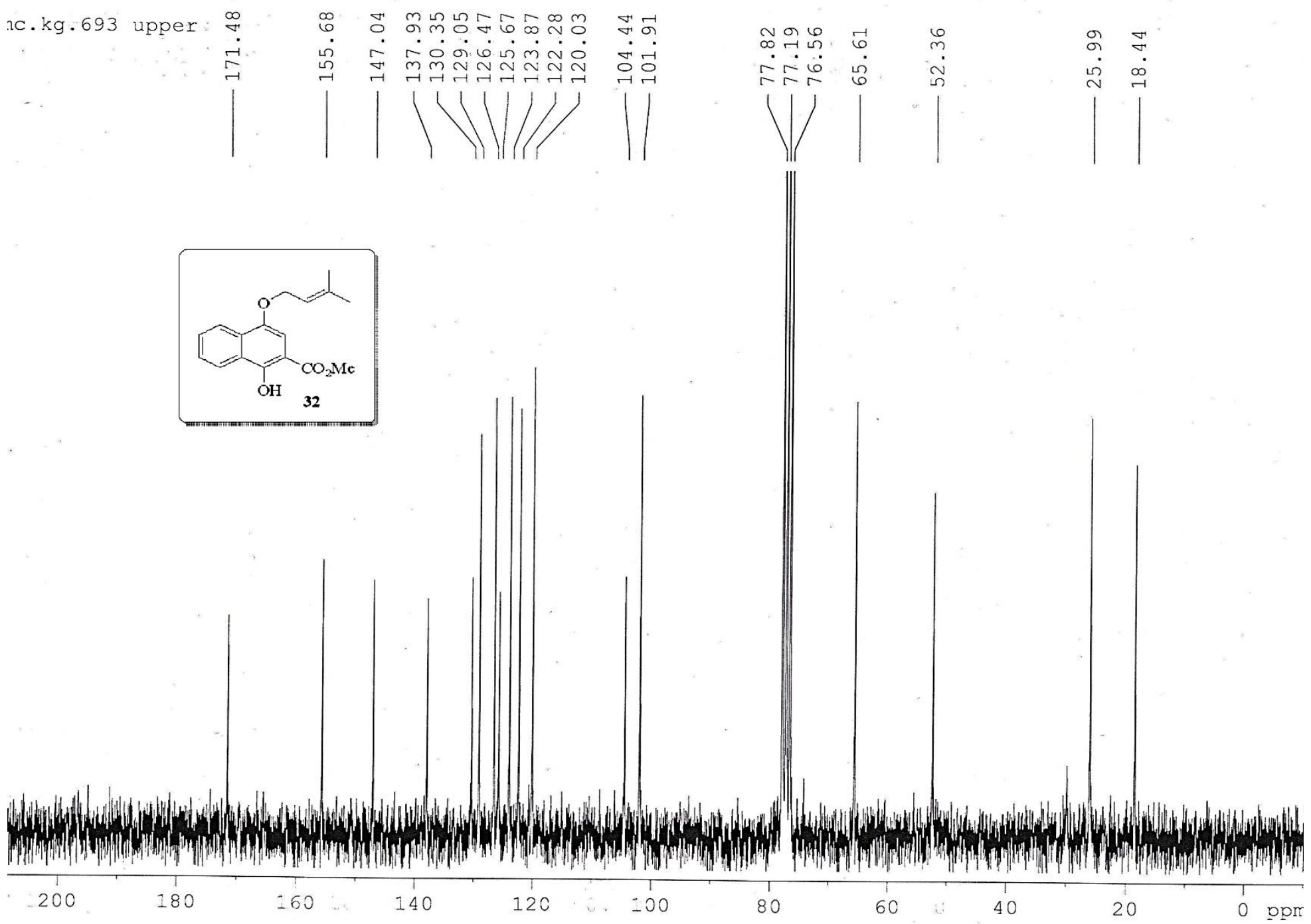


${ }^{1} \mathrm{H}$ NMR of compound 34a $\left(\mathrm{CDCl}_{3}, 400 \mathrm{MHz}\right)$

$\mathrm{dm} / \mathrm{kg} / 746-1 \mathrm{~h}$

$\underset{\mid}{\overrightarrow{6}}$
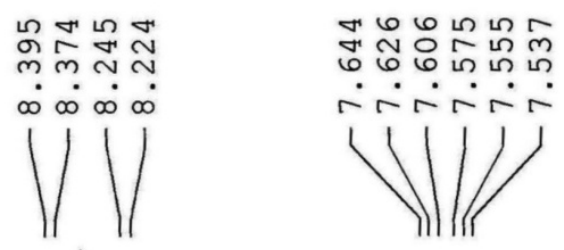

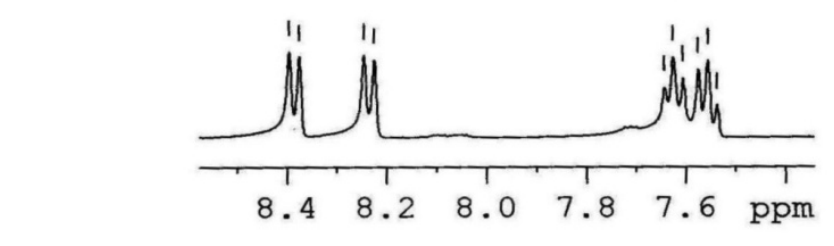

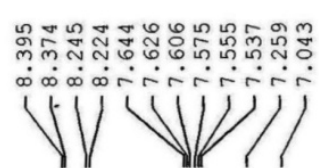
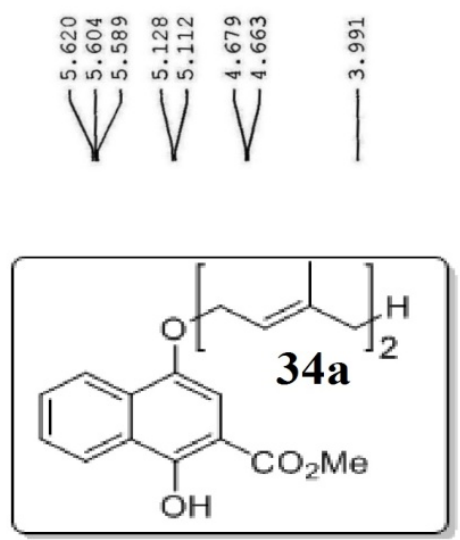

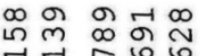
जं

1111

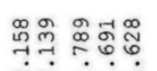

VIII
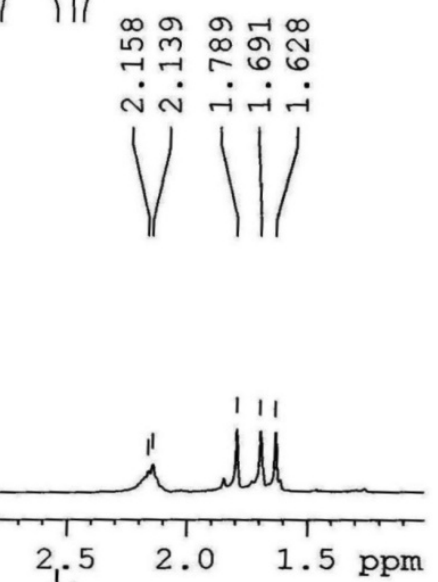
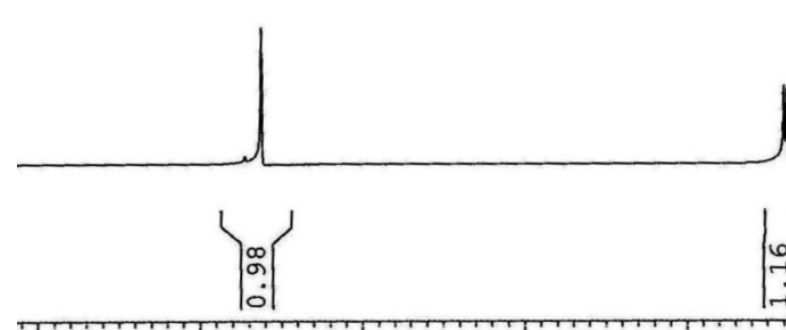

$13 \quad 12$

11

10
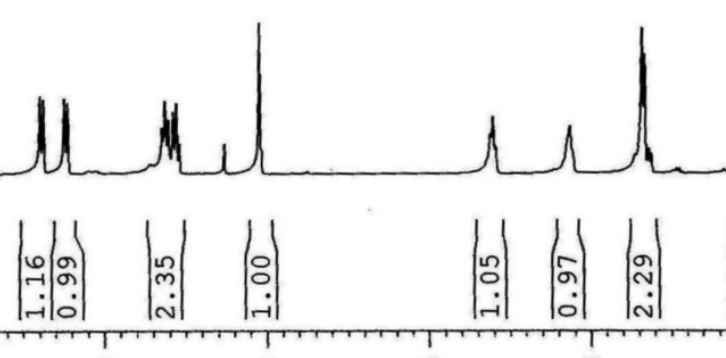

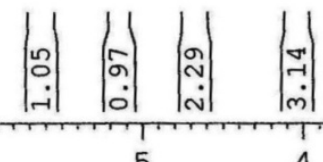

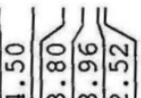

2

1

0 ppm 
${ }^{13} \mathrm{C}$ NMR of compound 34a $\left(\mathrm{CDCl}_{3}, 100 \mathrm{MHz}\right)$

the. ky. 140 doL spo.
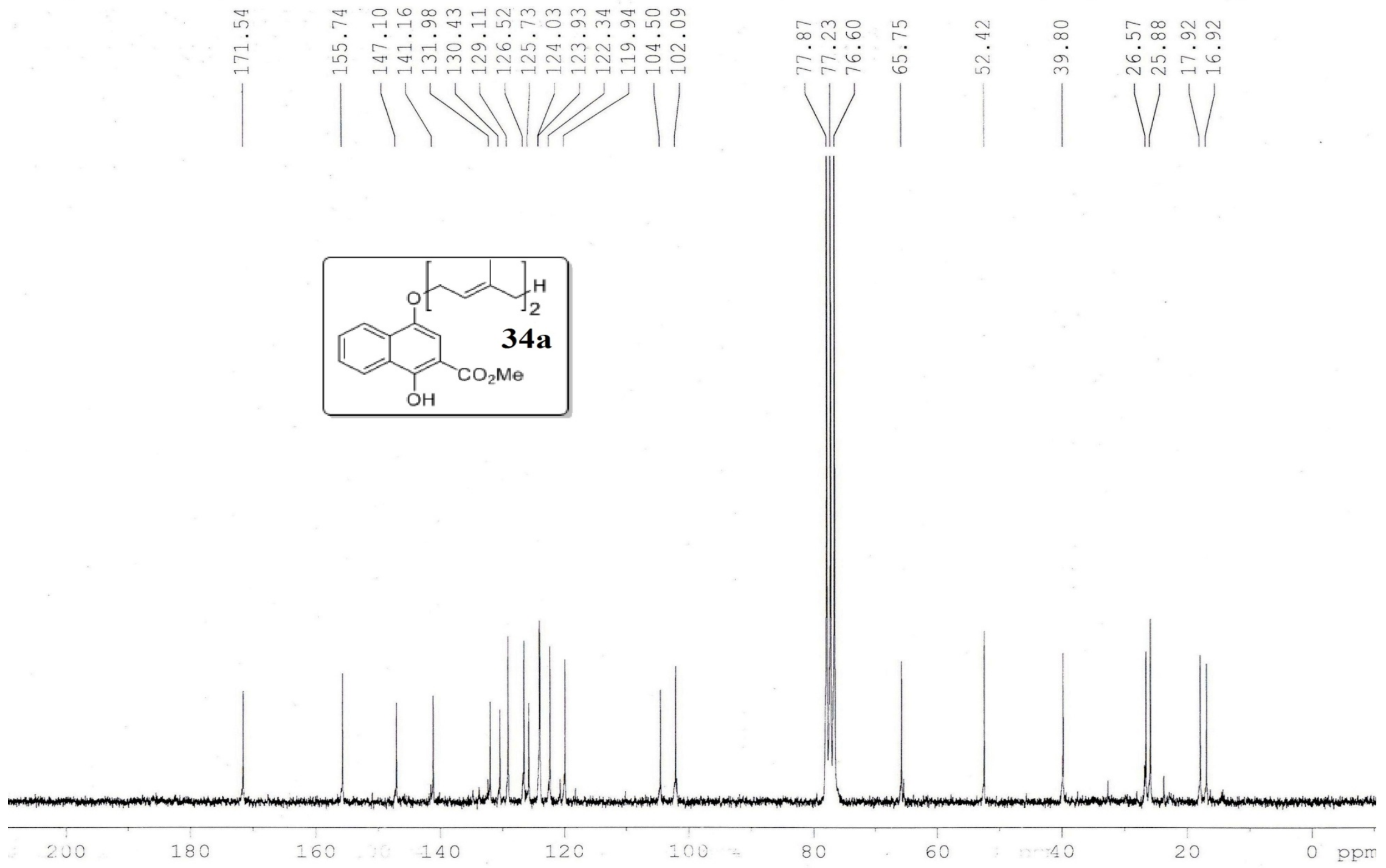
${ }^{1} \mathrm{H}$ NMR of compound $39\left(\mathrm{CDCl}_{3}, 600 \mathrm{MHz}\right)$

$\mathrm{dm}$

$/ \mathrm{sj} / 425 \mathrm{c}-1 \mathrm{~h}$

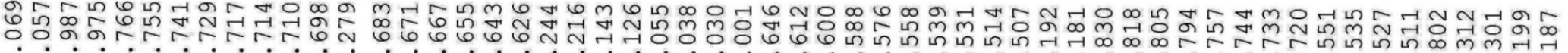
o ம

12

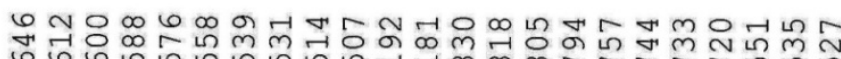

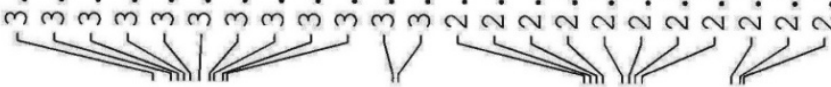
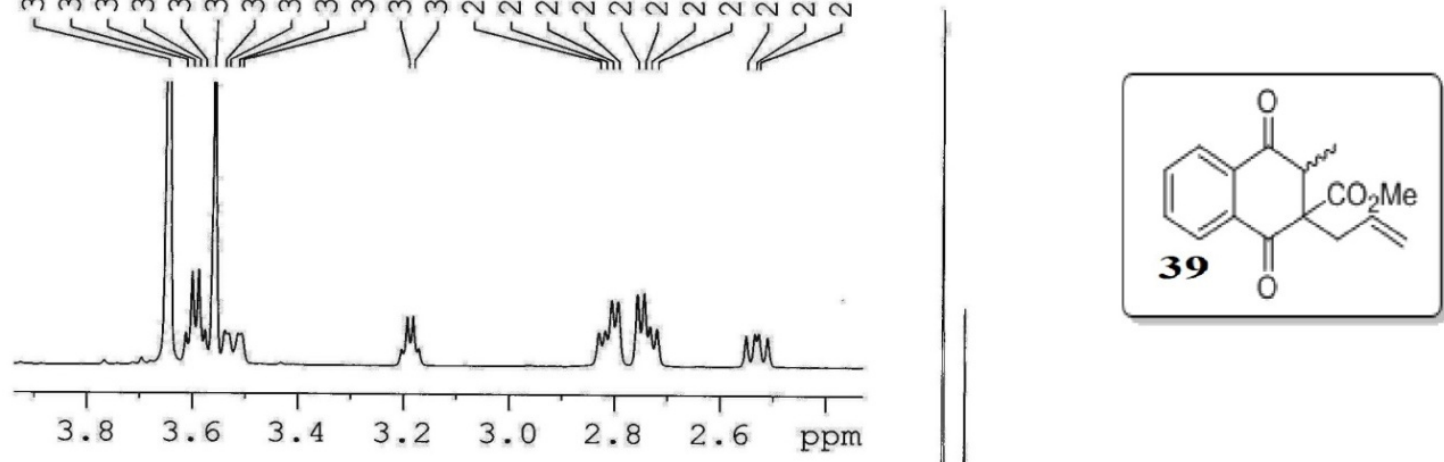

$\begin{array}{lllll}3.4 & 3.2 & 3.0 & 2.8 & 2.6\end{array}$

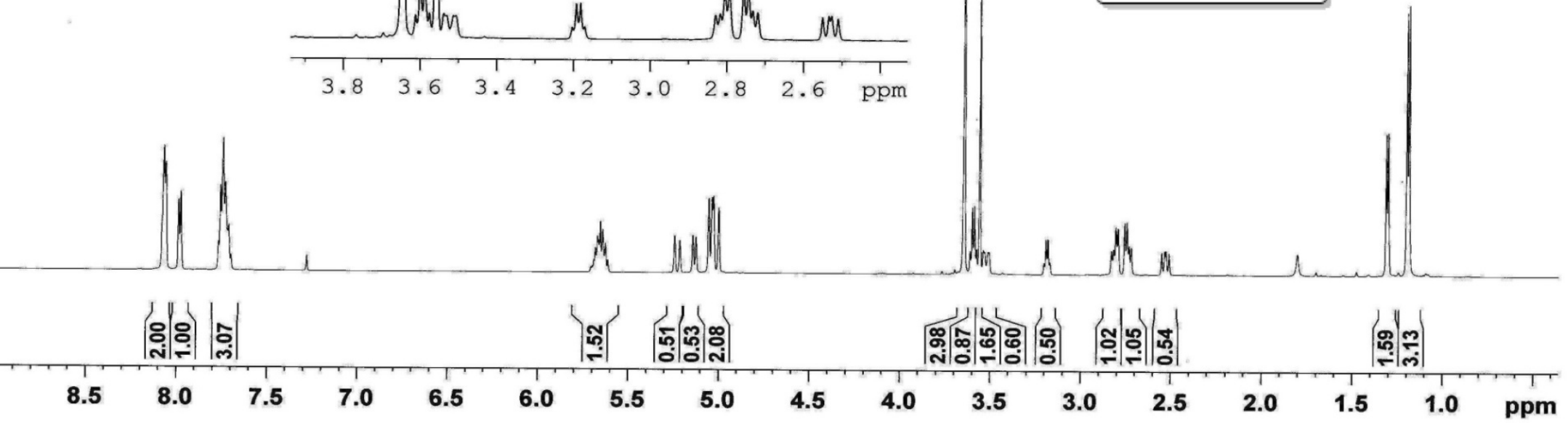


${ }^{1} \mathrm{H}$ NMR of compound $39\left(\mathrm{CDCl}_{3}, 600 \mathrm{MHz}\right)$

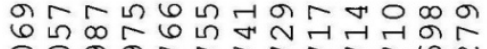
moriririririrar $\longrightarrow$

क人ิ

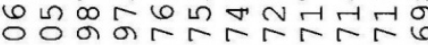

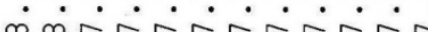
$\infty \pi+54505$ 1
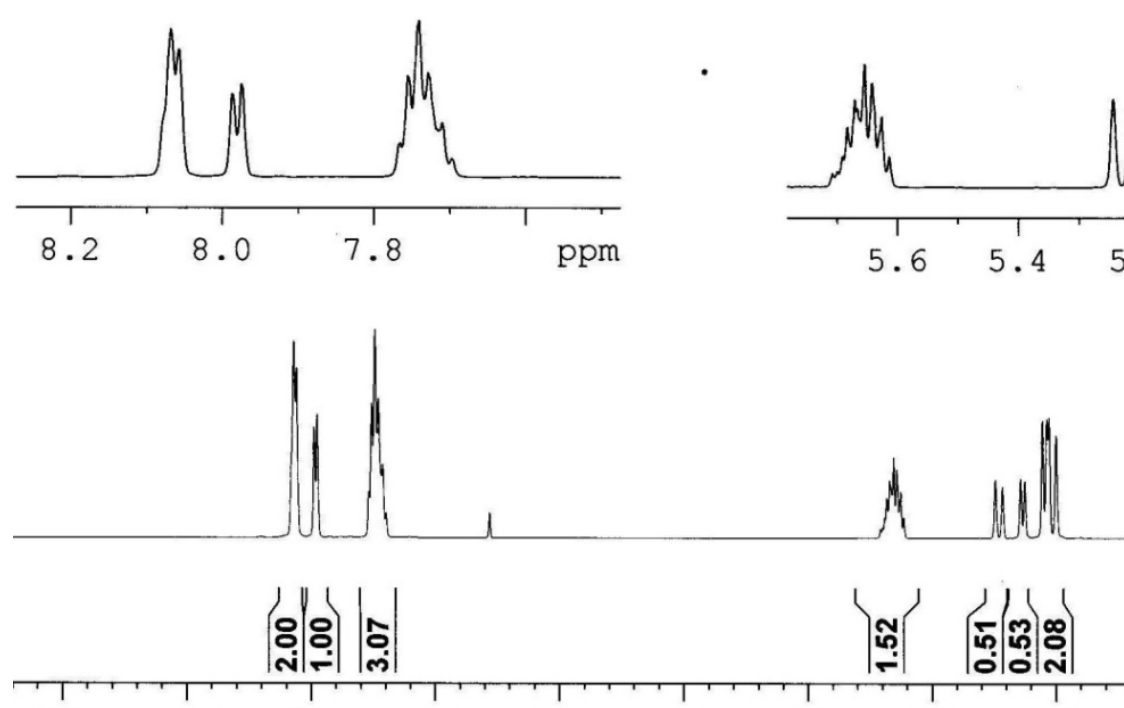

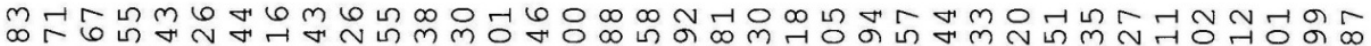
ம் ம் ம் ம் ம் ம் ம் ம் ம் ம் 31 l
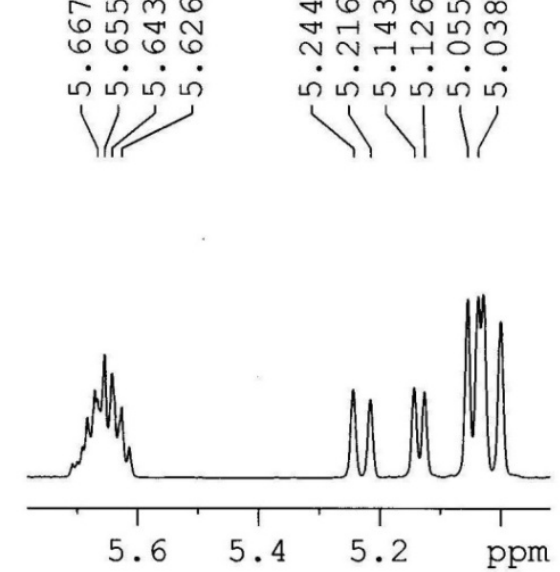

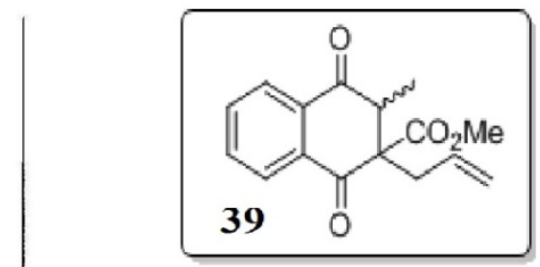

$M$ (1) 
${ }^{13} \mathrm{C}$ NMR of compound $39\left(\mathrm{CDCl}_{3}, 150 \mathrm{MHz}\right)$

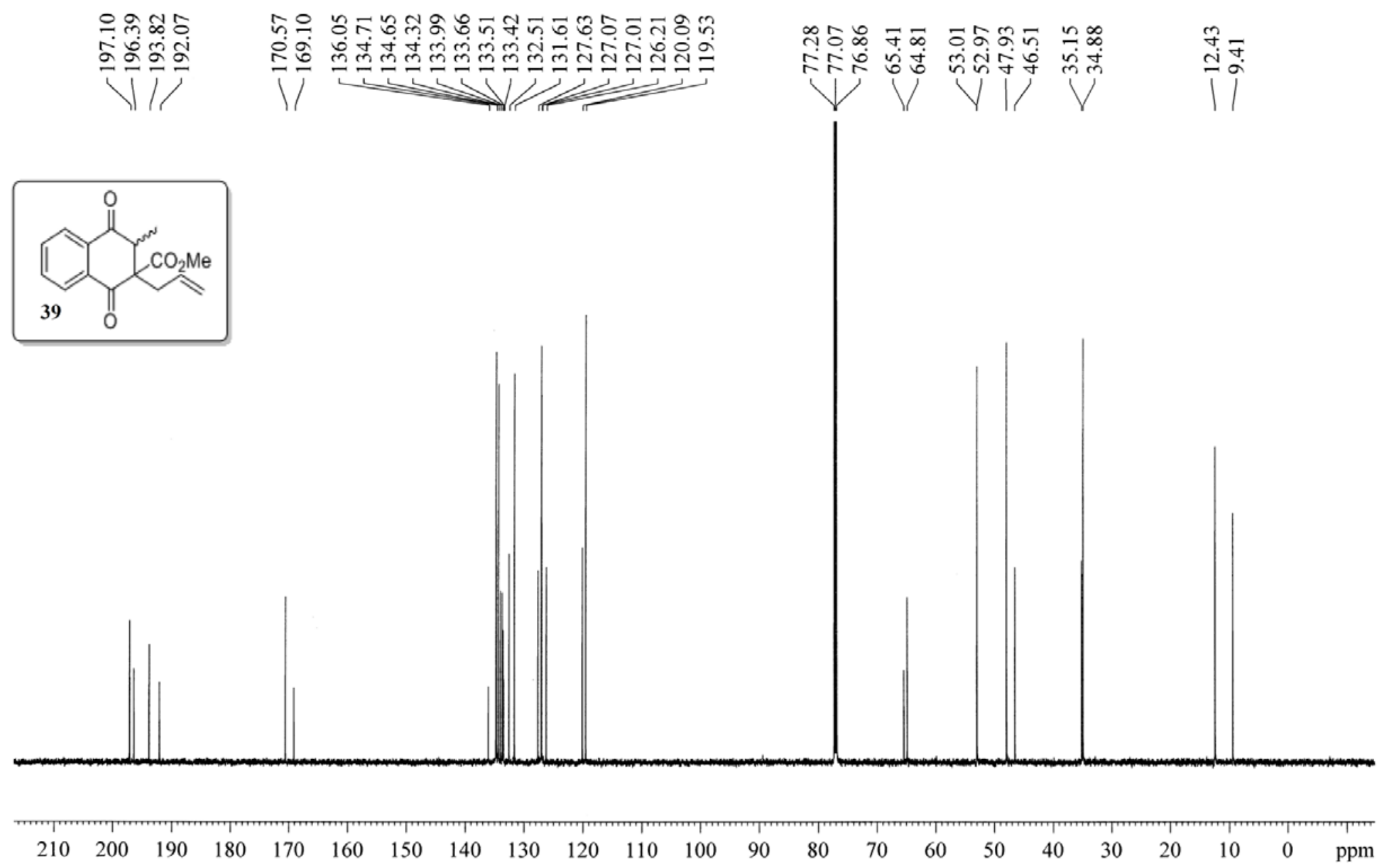


${ }^{1} \mathrm{H}$ NMR of compound $41\left(\mathrm{CDCl}_{3}, 600 \mathrm{MHz}\right)$

$\mathrm{dm} / \mathrm{sj} / 422 \mathrm{c}-1 \mathrm{~h}-\mathrm{f} 578$

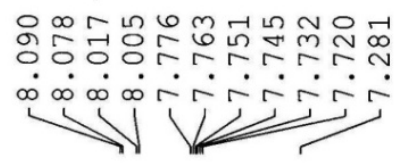

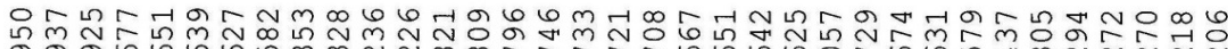

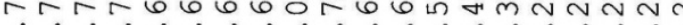

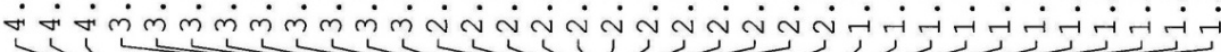

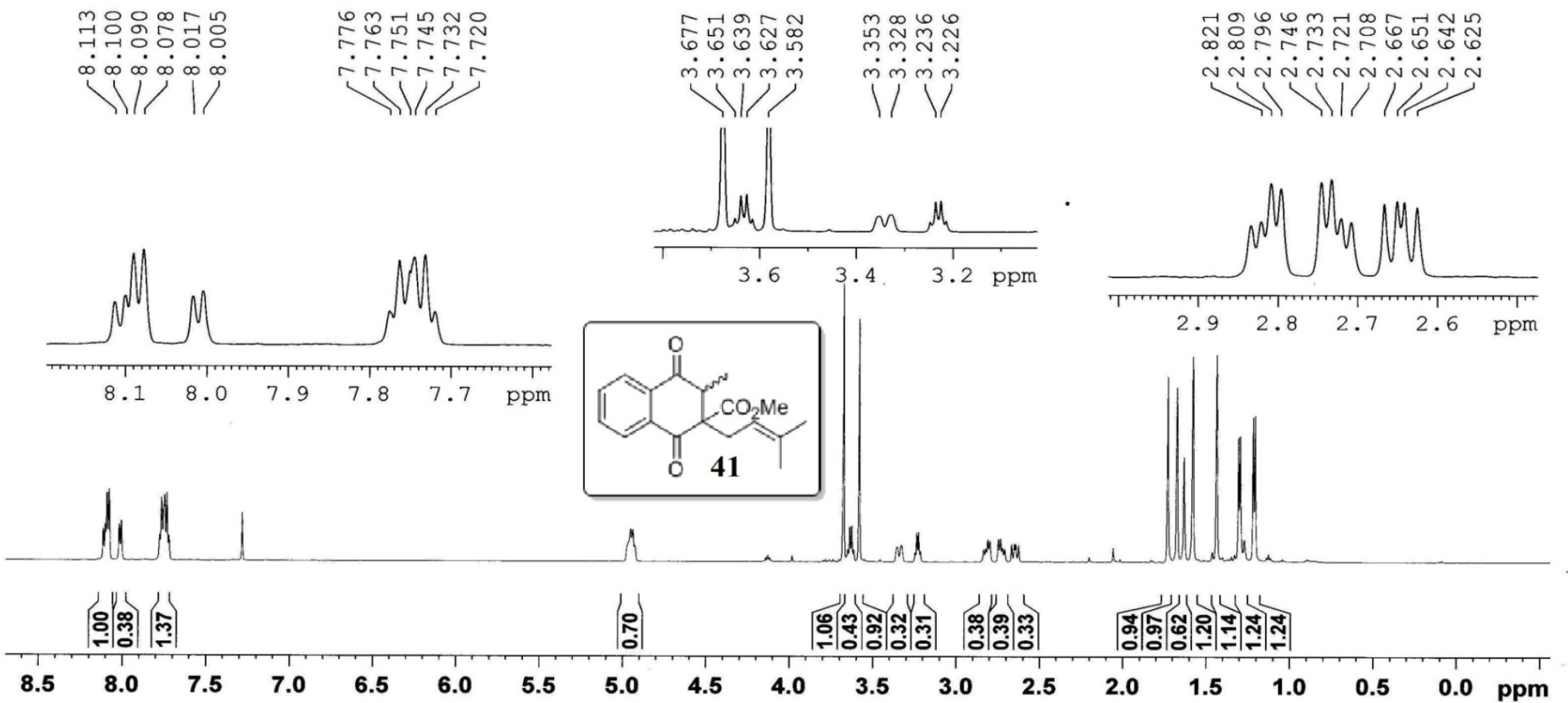


${ }^{13} \mathrm{C}$ NMR of compound $41\left(\mathrm{CDCl}_{3}, 150 \mathrm{MHz}\right)$

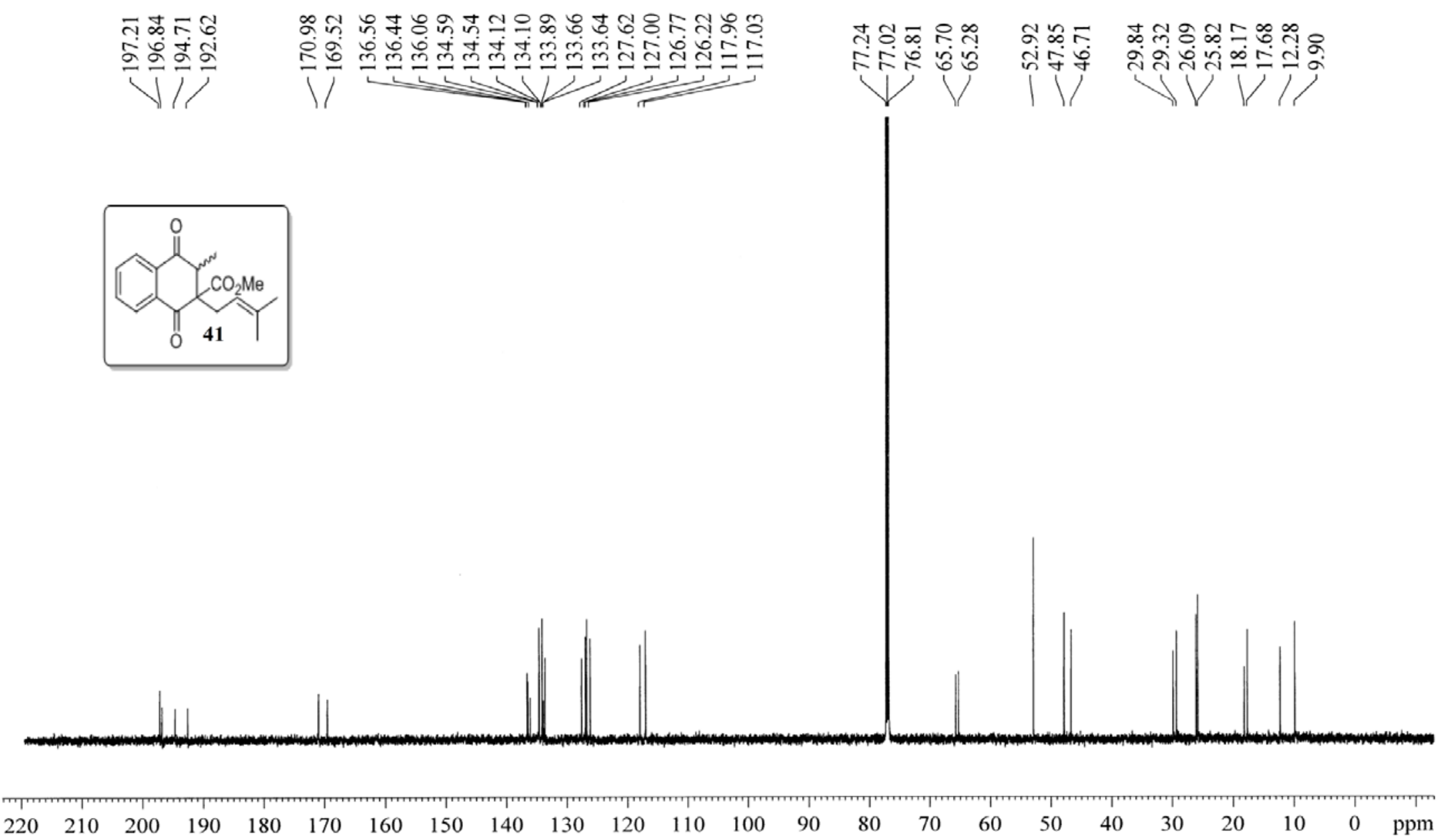


${ }^{1} \mathrm{H}$ NMR of compound $43\left(\mathrm{CDCl}_{3}, 600 \mathrm{MHz}\right)$

$\mathrm{dm} / \mathrm{sj} / 433-1 \mathrm{~h}-\mathrm{f} 649$

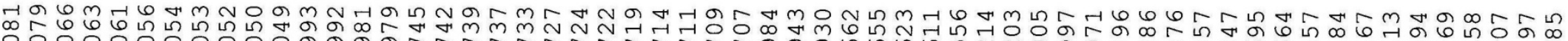

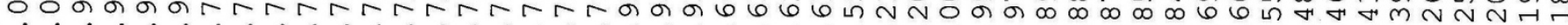

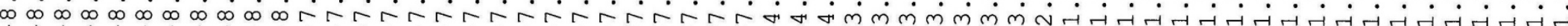
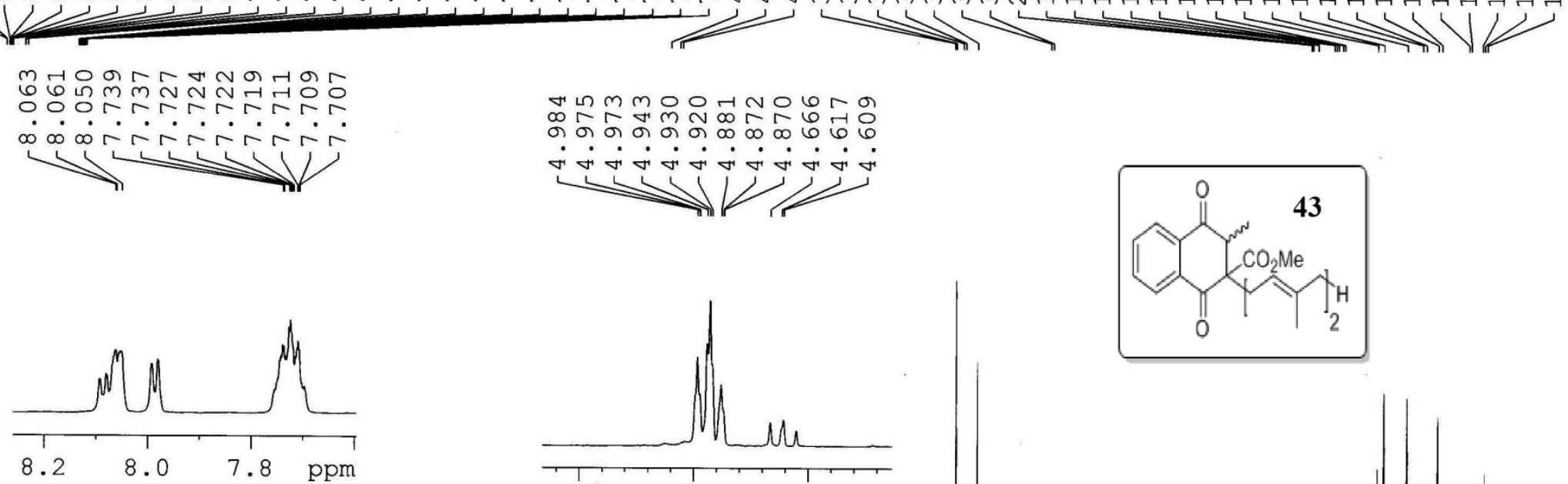

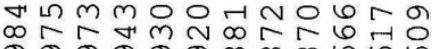
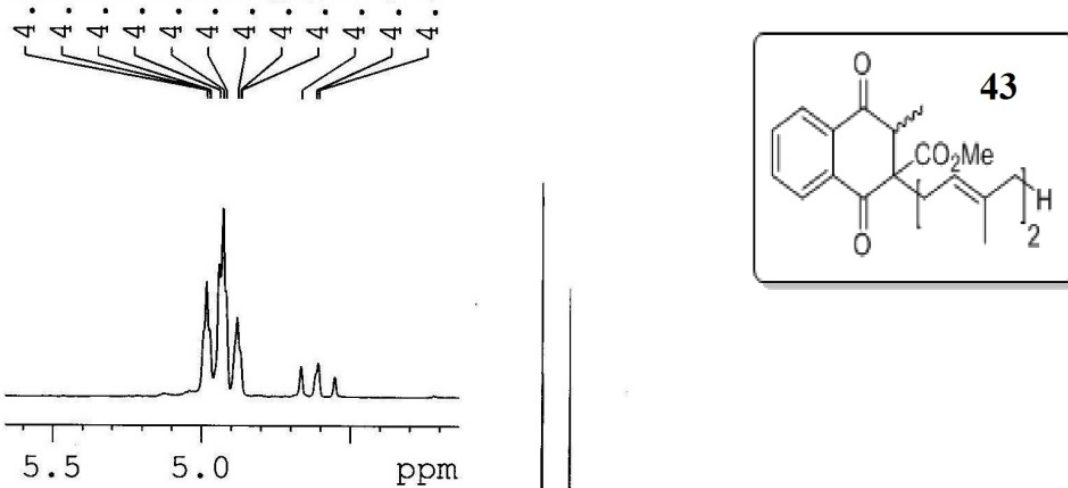

$8.2 \quad 8.0 \quad 7.8 \quad \mathrm{ppm}$
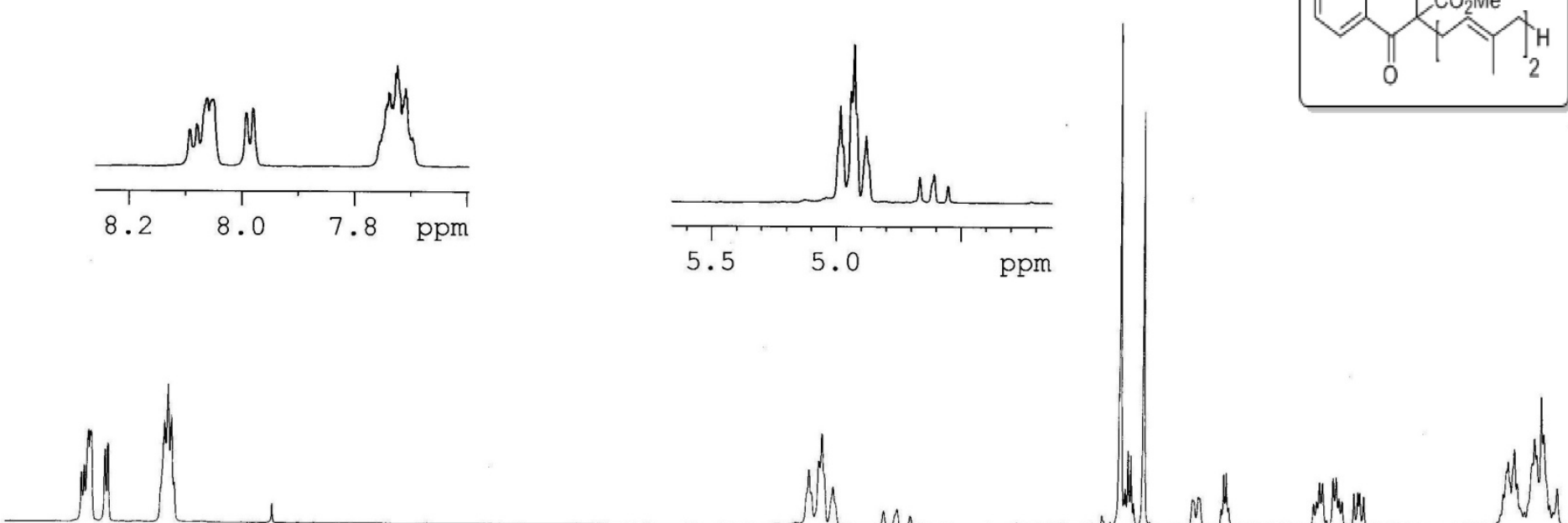

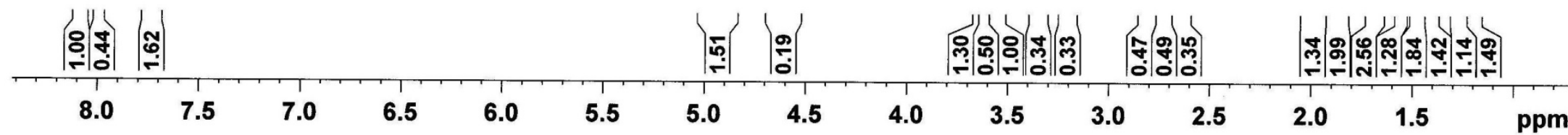


${ }^{1} \mathrm{H}$ NMR of compound $43\left(\mathrm{CDCl}_{3}, 600 \mathrm{MHz}\right)$

$\mathrm{dm} / \mathrm{sj} / 433-1 \mathrm{~h}-\mathrm{f} 649$

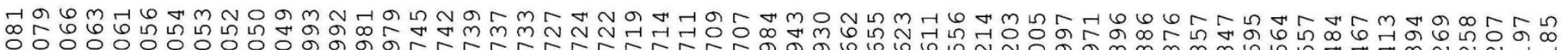

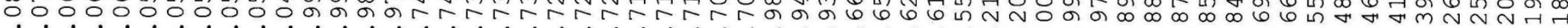

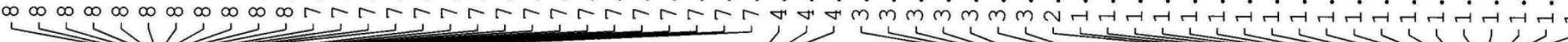

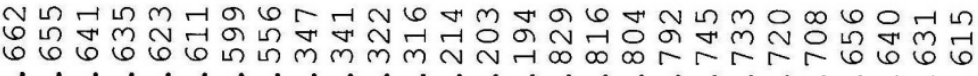

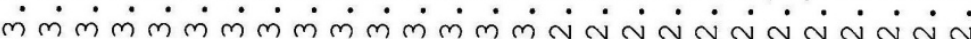
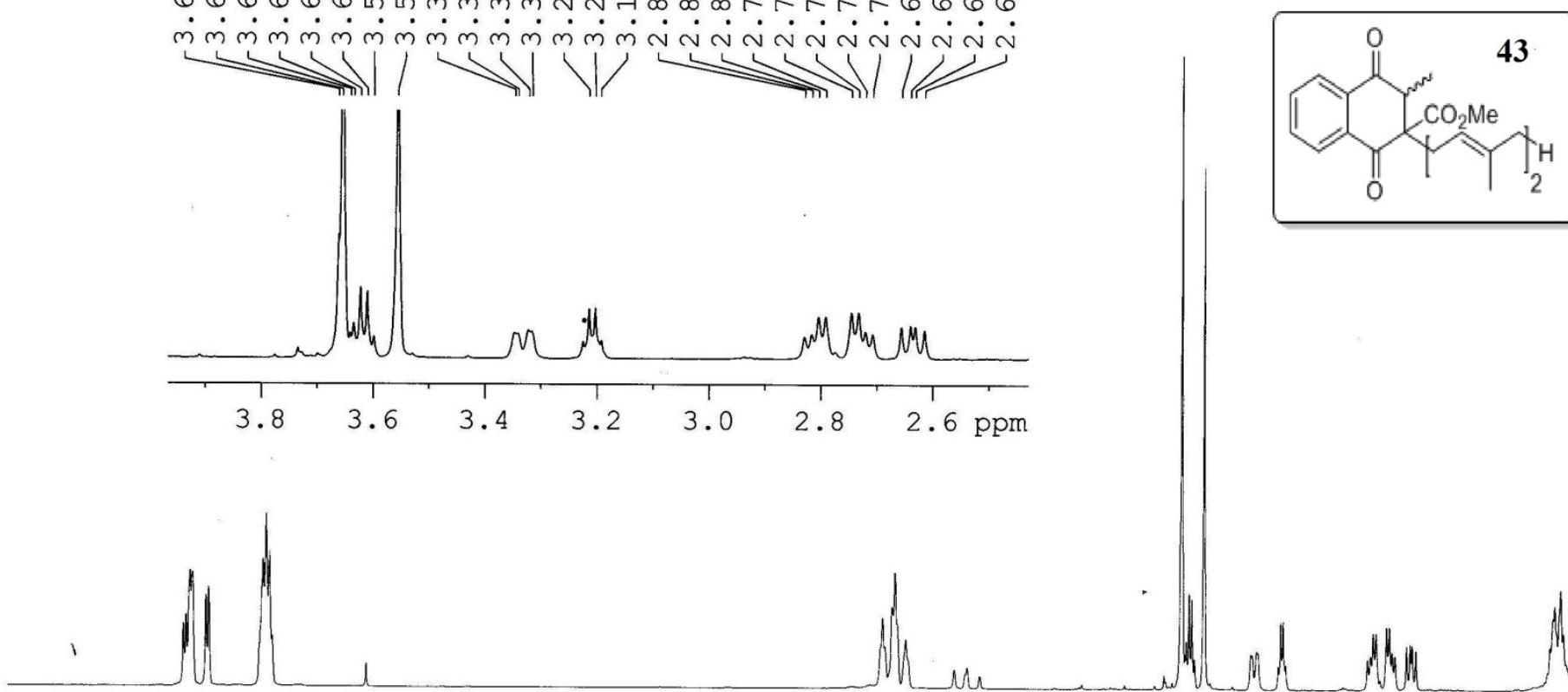

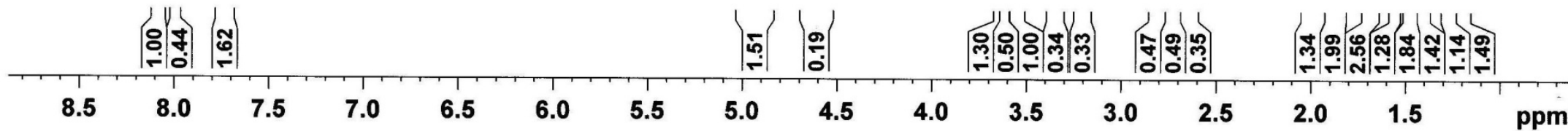




\section{${ }^{13} \mathrm{C}$ NMR of compound $43\left(\mathrm{CDCl}_{3}, 150 \mathrm{MHz}\right)$}

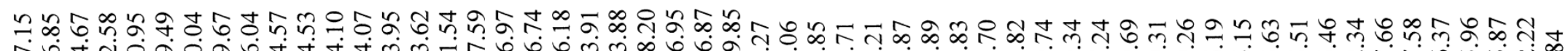

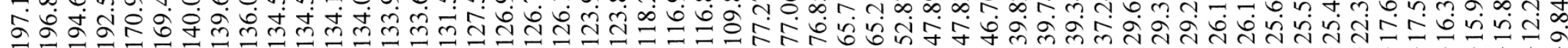
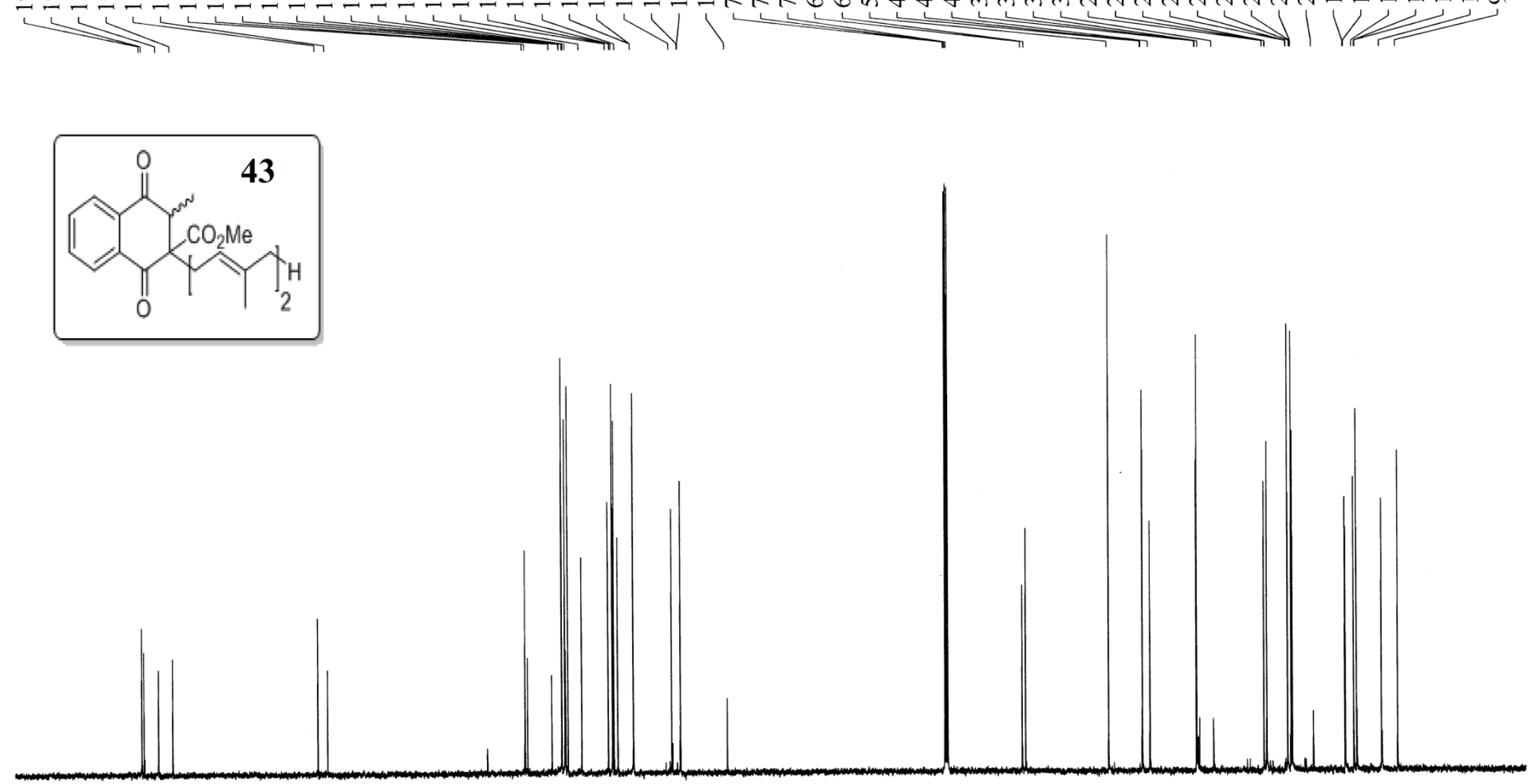
$210 \quad 200$
$190 \quad 180$
$170 \quad 160$
$150 \quad 140$
$130 \quad 120$
$90 \quad 80$
40
30
$20 \quad 10$
$0 \mathrm{ppm}$

$110 \quad 100$

$60 \quad 50$ 
${ }^{1}$ a) Fujii, F.; Shimizu, A.; Takeda, N.; Oguchi, K.; Katsurai, T.; Shirakawa, H.; Komai, M.; Kagechika, H.; Bioorg. Med. Chem. 2015, 23, 2344. b) Yamago,S.;Hashidume,M.;Yoshida,J. Tetrahedron2002, 58, 6805. b) Yamago,S.;Hashidume,M.;Yoshida,J. Tetrahedron2002, 58, 6805.

${ }^{2}$ Baraldi, P. G.; Guarneri, M.; Pollini, G. P.; Simoni, D.; Barco, A.; Benetti, S. J. Chem. Soc. Perkin Trans. 11984, 2501.

${ }^{3}$ Motoyoshiya, J.; Takagi, A.; Hirakawa, K.; Kakurai, T. J. Heterocyclic Chem. 1986, 23, 597.

${ }^{4}$ a) Mal, D.; Pahari, P.; Senapati, B. Tetrahedron Lett. 2005, 46, 2097. b) Yadav, J. S.; Reddy, B. V. S.; Swamy, T. Tetrahedron Lett. 2003, 44, 4861.

${ }^{5}$ a) Furumoto, T.; Hoshikuma, A. Phytochemistry, 2011, 72, 871. b) Araki, S.; Katsumura, N.; Butsugan, Y. J. Organomet. Chem. 1991, 415, 7.

${ }^{6}$ Syper, L.; Kloc, K.; Mlochowski, J. Tetrahedron 1980, 36, 123.

${ }^{7}$ Teitelbaum, A.; Scian, M.; Nelson, W.; Rettie, A. Synthesis 2015, 47, 944. 